Fields Institute Communications

Volume 60, 2011

\title{
l-Adic Étale Cohomology of PEL Type Shimura Varieties with Non-Trivial Coefficients
}

\author{
Elena Mantovan \\ Mathematics 253-37 \\ Caltech \\ Pasadena, CA 91125 USA \\ mantovanQcaltech.edu
}

\begin{abstract}
Given a Shimura datum $(G, h)$ of PEL type, let $p$ be an odd prime at which $G$ is unramified. In [13], we established a formula computing the $l$-adic cohomology of the associated Shimura varieties (regarded as a representation of the adelic points of $G$ and of the local Weil group at $p$ ) in terms of that of their local models at $p$ (the associated Rapoport-Zink spaces) and of the corresponding Igusa varieties. In this paper we extend those results (which are for cohomology with constant $l$-adic coefficients) to the general case of coefficients in a lisse étale sheaf attached to a finite dimensional $l$-adic representation of the group $G$.
\end{abstract}

\section{Introduction}

To any reductive group $G$ defined over a number field, one can associate a projective system of differentiable manifolds endowed with an action of $G\left(\mathbb{A}^{\infty}\right)$, the finite adelic points of $G$. Furthermore, to each algebraic representation $\rho$ of $G$ on a finite dimensional complex vector space, one can attach a local system $\mathfrak{L}_{\rho}$ on the corresponding manifolds and consider their complex cohomology spaces with coefficients in $\mathfrak{L}_{\rho}$ as representations of $G\left(\mathbb{A}^{\infty}\right)$. These complex cohomology spaces can then be interpreted in terms of automorphic forms on $G$. Moreover, for any cuspidal automorphic representation $\Pi$ of $G(\mathbb{A})$, satisfying certain "algebraic" conditions at infinity, there is a suitable choice of a representation $\rho$ such that $\Pi^{\infty}$ will appear in the associated cohomology spaces. For a certain class of $G$ 's, which was isolated by Shimura, these differential manifolds have a natural algebraic structure defined over a number field $E$. In most cases, the algebraic structure is obtained by interpreting the manifolds as classifying spaces over $\mathbb{C}$ for abelian varieties with additional structures. E.g., in the PEL case, the Shimura varieties arise as moduli spaces of abelian varieties endowed with polarizations, prescribed endomorphisms and level structures. Furthermore, to each algebraic representation $\xi$ of $G$ on a

2010 Mathematics Subject Classification. Primary 11G18, 11F70; Secondary 14G22, 14L05. Partially supported by the National Science Foundation under Grant DMS-0701310.

(C2011 American Mathematical Society 
finite dimensional $l$-adic vector space, one can attach a lisse étale $l$-adic sheaf $\mathcal{L}_{\xi}$ on the corresponding varieties and their l-adic étale cohomology groups with coefficients in $\mathcal{L}_{\xi}$ are naturally representations of $G\left(\mathbb{A}^{\infty}\right) \times \Gamma_{E}$, for $\Gamma_{E}$ the absolute Galois group of $E$. Again, the finite component of a cuspidal automorphic representation II of $G(\mathbb{A})$, satisfying certain algebraic conditions at infinity, will appear in some $l$-adic étale cohomology group for a suitable choice of $\xi$. A conjecture of Langlands predicts that the Galois representation associated to an "algebraic" cuspidal automorphic representation $\Pi$ of $G(\mathbb{A})$ under the (conjectural) global Langlands correspondences can be detected by looking at the $\Pi^{\infty}$-isotypic component $\Sigma_{\Pi}$ of these cohomology spaces. In view of the compatibility between global and local Langlands correspondences, one also expects that, for any prime number $p$, the restriction of $\Sigma_{\Pi}$ to $W_{E_{v}}$, the local Weil group at a prime $v \mid p$, depends only on the $p$-adic component of $\Pi$. This latter prediction is the main motivation for our work, which focus on the study of the $l$-adic étale cohomology groups of Shimura varieties of PEL type as representations of $G\left(\mathbb{A}^{\infty}\right) \times W_{E_{v}}$, for $v$ a prime of $E$ dividing a fixed prime number $p$ which we assume to be unramified for the group $G$. More precisely, in this paper we compute the $l$-adic étale cohomology groups of the Shimura varieties, with coefficients in $\mathcal{L}_{\xi}$ (for $\xi$ an irreducible finite dimensional $l$-adic representation of $G$ ), in terms of the $l$-adic étale cohomology groups of their local models, constructed by Rapoport and Zink, and those of the associated Igusa varieties. The latter are classifying spaces for abelian varieties in positive characteristic, whose defining moduli problems are closely related to those of the Shimura varieties. In the resulting formula, both the actions of the $p$-adic points of $G$ and of the local Weil group are trivial on the $l$-adic étale cohomology groups of the Igusa varieties but not on those of the Rapoport-Zink spaces. Moreover, only the $l$-adic étale cohomology groups of the Igusa varieties have coefficients in a local system depending on $\xi$ (namely, the pullback of $\mathcal{L}_{\xi}$ ) while those of the Rapoport-Zink spaces have trivial $l$-adic coefficients. These two facts make the formula heuristically compatible with Langlands' conjecture, in the sense explained above, and also with a conjecture of Kottwitz which predicts that the local Langlands correspondences at $p$ (for the groups $G$ 's in the class considered in this papers) are realized by the $l$-adic étale cohomology of the Rapoport-Zink spaces, with trivial coefficients. We remark that a special case of this formula, namely the case when $\xi$ is the trivial representation, was previously established in [13]. The goal of this paper is to generalized those results to any $\xi$. In view of the general philosophy, the case of $l$-adic étale cohomology with constant coefficients is too restrictive for most applications.

1.1 Outline. Our strategy closely follows that of [13], where the final formula (in the case of trivial $l$-adic coefficients) is deduced by results about the local geometry of the Shimura varieties at the chosen prime $v \mid p$. In particular, the formula in [13] can be regarded as an application of the appropriate Hochschild-Serre spectral sequence and Künneth formula, and reflects a description of (a stratification of) the Shimura varieties at $v$ as quotients by the action of a group of the products of their local models and Igusa varieties. Similarly, the formula with non-constant coefficients proved in this paper (and more precisely, its special case for $\xi$ the standard representation) reflects analogous statements about the local geometry at $v$ of the universal abelian scheme over the Shimura varieties (and its multi-self-products). In particular, we prove that the universal abelian schemes over the Shimura varieties at $v$ and those over the Igusa varieties become isogenous once pull-backed over the 
products of the Rapoport-Zink local models and Igusa varieties. We remark that while it would be possible to reproduce in this new context all of the constructions of [13], we chose not to. Instead, we preferred to deduce the new results from those in [13]. In Section 2, we recall results and constructions from the theory of integral models of Shimura varieties of PEL type, following [10] for the cases of good reduction and [13] for the general cases. In Section 3, we state the main theorem of this paper and outline a strategy for its proof. In Section 4, we show that the general case of this theorem can be deduced from a special case, namely the case of $\xi$ the standard representation. Finally, in Section 5, we prove the latter case. Throughout the paper, our notations follows those in [7] and [13].

\section{Shimura varieties of PEL type}

2.1 Integral models at unramified primes. In this section, we recall the definition of Shimura varieties of PEL type as moduli spaces for abelian varieties with additional structures. We focus in particular on the cases of good reduction at a chosen prime $p$. Our exposition follows [10] (Section 5, pp. 389-392) and we refer to it for details. A warning about notations: in certain cases the moduli spaces here described are not the canonical models of the corresponding Shimura varieties but disjoint unions of finitely many copies of them (see [10], Sec. 8, pp. 398-400). In the following, we will ignore this issue and refer to these moduli spaces as Shimura varieties.

2.1.1 We fix data of the following type:

- $B$ a finite dimensional simple algebra over $\mathbb{Q}$;

- * a positive involution on $B$ over $\mathbb{Q}$;

- $V$ a nonzero finitely generated left $B$-module;

- $\langle$, $\rangle$ a non degenerate $\mathbb{Q}$-valued $*$-hermitian alternating pairing on $V$.

To the above data, we associate an algebraic group $G$ over $\mathbb{Q}$ as follows. We define $G$ as the group of the $B$-linear automorphisms of $V$ which preserve the pairing $\langle$, up to scalar multiple, i.e. for any $\mathbb{Q}$-algebra $R$

$G(R)=\left\{(c, g) \in R^{\times} \times \operatorname{Aut}_{B \otimes_{\mathbb{Q}} R}\left(V \otimes_{\mathbb{Q}} R\right) \mid\langle g v, g w\rangle=c\langle v, w\rangle\right.$ for all $\left.v, w \in V \otimes_{\mathbb{Q}} R\right\}$.

In this paper we are occupied with the class of Shimura varieties associated with an algebraic group $G$ of this form. These varieties are called of PEL type. We remark that any group $G$ of this form is equipped with a canonical homomorphism $\nu: G \rightarrow \mathbb{G}_{m}$, which to a $B$-linear automorphism of $V$ preserving the pairing $\langle$,$\rangle up$ to scalar multiple associates the latter, i.e. for any $\mathbb{Q}$-algebra $R$ and any element $(c, g) \in G(R)$ we define $\nu(R)(c, g)=c$. Let $(G, h)$ be a Shimura datum associated with some data $(B, *, V,\langle\rangle$,$) as above. As usual, h$ denotes a conjugacy class of cocharacters $\mu_{h}: \mathbb{G}_{m} \rightarrow G_{\mathbb{C}}$ satisfying the appropriate positivity condition. Then, the Shimura field $E=E(G, h) \subset \mathbb{C}$, associated with the pair $(G, h)$, is the field of definition of the isomorphism class of the complex representation $V_{1}$ of $B$, where $V_{1}$ is the subspace of $V_{\mathbb{C}}$ on which $\mu_{h}(z)$ acts as $z$. The Shimura varieties attached to the datum $(G, h)$ have a natural structure of algebraic varieties defined over $E$. More precisely, let $\mathbb{A}^{\infty}$ denote the ring of the finite adeles of $\mathbb{Q}$, and $K$ an open compact subgroup of $G\left(\mathbb{A}^{\infty}\right)$. For $K$ sufficiently small, the associated Shimura variety $\mathrm{Sh}_{K}$ is a smooth quasi-projective scheme defined over $E$, and has a moduli interpretation as a classifying space for abelian varieties. As $K$ varies, the varieties $\mathrm{Sh}_{K}$ form a projective system, naturally endowed with an action of $G\left(\mathbb{A}^{\infty}\right)$. We 
refer to [10] for the definition of PEL type moduli problems for abelian varieties over the reflex field $E$. Here, we focus on the cases of good reduction at a chosen prime $p$, and introduce the corresponding moduli problems over $\mathcal{O}_{E,(p)}$, the localization at $p$ of the ring of integers of $E$.

2.1.2 Let $p$ be an odd prime. We assume that the PEL data $(B, *, V,\langle\rangle$, satisfy the following three conditions:

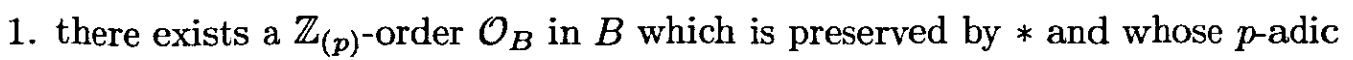
completion is a maximal order in $B_{\mathbb{Q}_{p}}$;

2. there exists a lattice $\Lambda$ in $V_{\mathbb{Q}_{p}}$ which is self-dual for $\langle$,$\rangle and is preserved by$ $\mathcal{O}_{B}$

3. (unramified hypothesis) $B_{\mathbb{Q}_{p}}$ is a product of matrix algebras over unramified extensions of $\mathbb{Q}_{p}$.

Under the above conditions, the group $G$ has an hyperspecial maximal compact subgroup $K_{p, 0}$ of $G\left(\mathbb{Q}_{p}\right)$, namely the stabilizer of $\Lambda$. In the following, we write $G\left(\mathbb{Z}_{p}\right)=K_{p, 0}, G\left(\mathbb{Q}_{p}\right)^{\dagger}=\left\langle G\left(\mathbb{Z}_{p}\right), p\right\rangle$ for the subgroup of $G\left(\mathbb{Q}_{p}\right)$ generated by $G\left(\mathbb{Z}_{p}\right)$ and multiplication by $p$ on $V$, and $G\left(\mathbb{A}^{\infty}\right)^{\dagger}=G\left(\mathbb{A}^{\infty, p}\right) \times G\left(\mathbb{Q}_{p}\right)^{\dagger}$. We also write $\mathcal{O}_{B_{Q_{p}}}$ for the $p$-adic completion of $\mathcal{O}_{B}$. Finally, we choose a basis $\alpha_{1}, \ldots \alpha_{t}$ for the

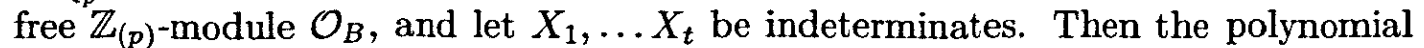
$\operatorname{det}\left(X_{1} \alpha_{1}+\cdots+X_{t} \alpha_{t} ; V_{1}\right)$ is homogeneous of degree $\operatorname{dim}_{\mathbb{C}}\left(V_{1}\right)$, with coefficients in $\mathcal{O}_{E,(p)}$. We restrict our attention to open compact subgroups $K$ of $G(\mathbb{A})$ of the form $K=K^{p} K_{p, 0}$, where $K^{p}$ is an open compact subgroup of $G\left(\mathbb{A}^{\infty, p}\right)$. We remark that if $K^{p}$ is sufficiently small, then so is $K$. We assume $K^{p}$ is sufficiently small. Under the above conditions, the Shimura varieties $\mathrm{Sh}_{K^{p} K_{p, 0}}$ admit integral models $S_{K^{p}}$ over $\mathcal{O}_{E,(p)}$ satisfying the following properties. For all $K^{p}$, the schemes $S_{K^{p}}$ are smooth and quasi-projective over $\mathcal{O}_{E,(p)}$, and as $K^{p}$ varies they form a projective system endowed with an action of $G\left(\mathbb{A}^{\infty, p}\right)$. Moreover, there exist canonical isomorphisms $\operatorname{Sh}_{K^{p} K_{p, 0}} \cong S_{K^{p}} \times_{\operatorname{Spec}\left(\mathcal{O}_{E,(p)}\right)} \operatorname{Spec}(E)$ which form a compatible system as the level $K^{p}$ varies, and are equivariant for the action of $G\left(\mathbb{A}^{\infty, p}\right)$. The schemes $S_{K^{p}}$ have a moduli interpretation as classifying spaces for abelian varieties. We recall their definition. To any open compact subgroup $K^{p} \subset G\left(\mathbb{A}^{\infty, p}\right)$, we associate a set-valued contravariant functor $\mathcal{F}_{K^{p}}$ on the category of locally Noetherian $\mathcal{O}_{E,(p)}$-schemes. It suffices to define $\mathcal{F}_{K^{p}}(S)$ for $S$ connected, in which case we first define $\mathcal{F}_{K^{p}}(S)=\mathcal{F}_{K^{p}}(S, s)$ for a choice of a geometric point $s \in S$, and then observe that such set is independent on the choice of $s \in S$. For any pair $(S, s)$, we define $\mathcal{F}_{K^{p}}(S, s)$ as the set of equivalence classes of quadruples $\left(A, \lambda, i, \bar{\eta}^{p}\right)$ where:

- $A$ is an abelian scheme over $S$;

- $\lambda: A \rightarrow A^{\vee}$ is a prime-to- $p$ polarization;

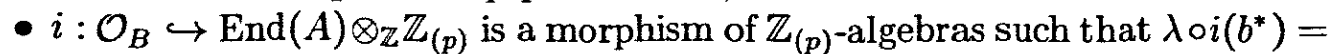
$i(b)^{\vee} \circ \lambda$, and $\operatorname{det}\left(X_{1} \alpha_{1}+\cdots+X_{t} \alpha_{t} ; \operatorname{Lie}(A)\right)=\operatorname{det}\left(X_{1} \alpha_{1}+\cdots+X_{t} \alpha_{t} ; V_{1}\right)$ (the latter is an equality of homogeneous polynomial with coefficients in the global sections of $\mathcal{O}_{S}$, known as the determinant condition);

- $\bar{\eta}^{p}$ is a $\pi_{1}(S, s)$-invariant $K^{p}$-orbit of isomorphisms of $B \otimes_{\mathbb{Q}} \mathbb{A}^{\infty, p}$-modules $\eta^{p}: V \otimes_{\mathbb{Q}} \mathbb{A}^{\infty, p} \rightarrow V^{p} A_{s}$ which takes the pairing $\langle$,$\rangle on V \otimes_{\mathbb{Q}} \mathbb{A}^{\infty, p}$ to a $\left(\mathbb{A}^{\infty, p}\right)^{\times}$-scalar multiple of the $\lambda$-Weil pairing. (Here, $V^{p} A_{s}$ denotes the Tate space of $A_{s}$ away from $p$ ).

Two quadruples $\left(A, \lambda, i, \bar{\eta}^{p}\right)$ and $\left(A^{\prime}, \lambda^{\prime}, i^{\prime},\left(\bar{\eta}^{p}\right)^{\prime}\right)$ are equivalent if there exists a prime-to- $p$ isogeny $\beta: A \rightarrow A^{\prime}$ which takes $\lambda$ to a $\mathbb{Z}_{(p)}^{\times}$-multiple of $\lambda^{\prime}, i$ to $i^{\prime}$ and $\bar{\eta}$ 
to $\left(\bar{\eta}^{p}\right)^{\prime}$. For $K^{p}$ sufficiently small, the functor $\mathcal{F}_{K^{p}}$ on the category of locally Noetherian $\mathcal{O}_{E,(p)}$-schemes is represented by a smooth quasi-projective $\mathcal{O}_{E,(p)}$-scheme. For each $K^{p}$ sufficiently small, the integral model $S_{K^{p}}$ is defined as the $\mathcal{O}_{E,(p)^{-}}$ scheme representing $\mathcal{F}_{K^{p}}$ ([10], Sec. 5, p. 391).

2.1.3 We now focus on the problem of defining integral models for $\mathrm{Sh}_{K}$, in the case when the level $K$ is not maximal at $p$. We consider exclusively the case of $K$ of the form $K^{p} K_{p}$ where $K^{p}$ is any sufficiently small open compact subgroup of $G\left(\mathbb{A}^{\infty, p}\right)$ and $K_{p}$ is an open compact subgroup of $G\left(\mathbb{Q}_{p}\right), K_{p} \subset K_{p, 0}$, of the form

$$
K_{p}=K_{p, m}=\left\{g \in K_{p, 0} \mid g_{\Lambda} \equiv 1 \quad \bmod p^{m} \Lambda\right\},
$$

for some integer $m \geq 0$. As $m$ varies, the subgroups $K_{p, m}$ form a fundamental system of open neighborhoods of the identity in $G\left(\mathbb{Q}_{p}\right)$, and similarly, as both $m$ and $K^{p}$ vary, the subgroups $K=K^{p} K_{p, m}$ describe a fundamental system of open compact subgroups of $G\left(\mathbb{A}^{\infty}\right)$. Thus, it is without loss of generality that through the rest of this paper we assume the open compact subgroups $K$ of $G\left(\mathbb{A}^{\infty}\right)$ to be of this form. For any such $K, K=K^{p} K_{p}$, we define the corresponding integral model of $S_{K}$ as a $S_{K^{p}}$-scheme, and denote it by $S_{K}$. The key ingredient is Katz and Mazur's notion of a full set of sections for finite flat schemes ([9], Section 1.8.2, p.33). Details and proofs of the construction can be found in [13] (Section 6, pp. 597-601). For any $m \geq 1$, and $K=K^{p} K_{p, m}$, we define $S_{K}$ as the $S_{K^{p} \text {-scheme }}$ representing the following functor $\mathcal{S}_{m}$. Let $A$ denote the universal abelian scheme over $S_{K^{p}}$. We write $A\left[p^{m}\right]$ for its $p^{m}$-torsion subgroup, $A\left[p^{\infty}\right]$ for its $p$-divisible part, and $\mu_{p^{m}}$ for the group scheme of the $p^{m}$-th roots of unity. We recall that the additional structures on $A$ induce corresponding structures on the Barsotti-Tate group $A\left[p^{\infty}\right]$, namely (in our case) a quasi-polarization (which we denote by $\ell$ ) and

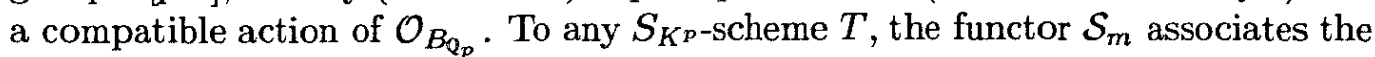
set of pairs $\left(\alpha_{0}, \alpha\right)$ where $\alpha_{0}: p^{-m} \mathbb{Z} / \mathbb{Z} \rightarrow \mu_{p^{m}}(T)$ and $\alpha: p^{-m} \Lambda / \Lambda \rightarrow A\left[p^{m}\right](T)$ are two group homomorphisms satisfying the conditions:

1. $\left\{\alpha_{0}(x) \mid x \in p^{-m} \mathbb{Z} / \mathbb{Z}\right\}$ is a full set of sections of $\mu_{p^{m}, T} / T$;

2. $\left\{\alpha(x) \mid x \in p^{-m} \Lambda / \Lambda\right\}$ is a full set of sections of $A\left[p^{m}\right]_{T} / T$;

3. $\alpha$ is $\mathcal{O}_{B_{Q_{p}}}$-equivariant;

4. the following diagram commutes

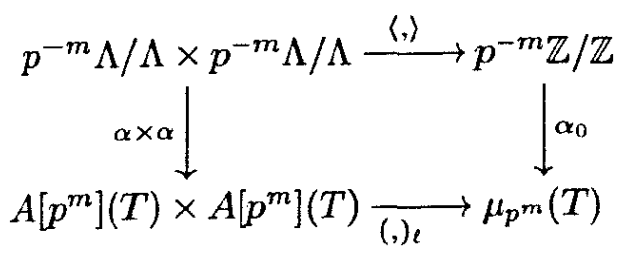

(here, with abuse of notations $\langle$,$\rangle denotes the pairing on p^{-m} \Lambda / \Lambda$ induced by that on $\Lambda$, and $(,)_{\ell}$ is the $\ell$-Weil pairing on $A\left[p^{m}\right](T)$ ).

For any $m \geq 1$, the functor $\mathcal{S}_{m}$ is representable. The representing scheme $S_{K}$ is finite over $S_{K^{p}}$, and naturally endowed with an action of $G\left(\mathbb{Z}_{p}\right)$, trivial on $K_{p, m}$. For $m=0$, we write $S_{K}=S_{K^{p}}$. As $K$ varies, the schemes $S_{K}$ form a projective system naturally endowed with an action of $G\left(\mathbb{A}^{\infty}\right)^{\dagger}=G\left(\mathbb{A}^{\infty, p}\right) \times G\left(\mathbb{Q}_{p}\right)^{\dagger}$. Moreover, for each $K$, there is a canonical isomorphism $\operatorname{Sh}_{K} \cong S_{K} \times{ }_{\operatorname{Spec}\left(\mathcal{O}_{E,(p)}\right)} \operatorname{Spec}(E)$, such that, as $K$ varies, they form compatible system which is $G\left(\mathbb{A}^{\infty}\right)^{\dagger}$-equivariant with respect to the natural action of $G\left(\mathbb{A}^{\infty}\right)$ on the Shimura varieties. We point out that in general the action of the whole $G\left(\mathbb{A}^{\infty}\right)$ on the Shimura varieties does not 
extend to the above integral models. On the other hand, for a slightly more general class of integral models, the associated correspondences do ([13], Proposition 17, p. 600 ).

2.2 Cohomology and vanishing cycle sheaves. Let $l$ be a prime number, $l \neq p$. Any continuous $l$-adic representation of $G\left(\mathbb{A}^{\infty}\right)$ gives rise to lisse étale $l$ adic sheaves $\mathcal{F}_{K}$ over $\mathrm{Sh}_{K}$, for all sufficiently small open compact subgroups $K$ of $G\left(\mathbb{A}^{\infty}\right)([10]$, Section 6, pp. 392-393; or [7], Section III.2, pp. 94-105). We apply this construction to $\xi$ an algebraic finite dimensional $l$-adic representation of $G$.

2.2.1 Let $\xi$ be an algebraic representation of $G$ on a finite dimensional $\mathbb{Q}_{l}^{\text {ac }}$ vector space $W_{\xi}$, and denote the corresponding sheaves on the Shimura varieties by $\mathcal{L}_{\xi}=\mathcal{L}_{\xi, K}$. As $K$ varies, the sheaves $\mathcal{L}_{\xi, K}$ form a compatible system, naturally endowed with an action of $G\left(\mathbb{A}^{\infty}\right)$. More precisely, for each $\gamma \in G\left(\mathbb{A}^{\infty}\right)$ and level $K$, let $K_{\gamma}=K \cap \gamma K \gamma^{-1}, f: \mathrm{Sh}_{K_{\gamma}} \rightarrow \mathrm{Sh}_{K}$ denote the natural projection between Shimura varieties corresponding to the inclusion $K_{\gamma} \subset K$, and $\gamma: \mathrm{Sh}_{K_{\gamma}} \rightarrow$ $\mathrm{Sh}_{\gamma^{-1} K_{\gamma \gamma}} \rightarrow \mathrm{Sh}_{K}$ the composition of the action of $\gamma$ with the natural projection between Shimura varieties. Then there is a canonical isomorphism between the pullbacks $\gamma^{*} \mathcal{L}_{\xi, K}$ and $f^{*} \mathcal{L}_{\xi, K}$ over $\mathrm{Sh}_{K_{\gamma}}$ ([10], Section 6, pp. 392-393). It follows that, for each $i \geq 0$, the $\mathbb{Q}_{l}^{\text {ac }}$-spaces

$$
H_{c}^{i}\left(\mathrm{Sh}, \mathcal{L}_{\xi}\right)=\lim _{K} H_{c}^{i}\left(\mathrm{Sh}_{K} \times_{E} E^{\mathrm{ac}}, \mathcal{L}_{\xi, K}\right)
$$

have a canonical structure of $G\left(\mathbb{A}^{\infty}\right) \times \mathrm{Gal}\left(E^{\mathrm{ac}} / E\right)$-modules. In fact, they are admissible/continuous $G\left(\mathbb{A}^{\infty}\right) \times \operatorname{Gal}\left(E^{\mathrm{ac}} / E\right)$-modules. We write

$$
H_{c}\left(\mathrm{Sh}, \mathcal{L}_{\xi}\right)=\sum_{i}(-1)^{i} H_{c}^{i}\left(\mathrm{Sh}, \mathcal{L}_{\xi}\right) \in \operatorname{Groth}\left(G\left(\mathbb{A}^{\infty}\right) \times \operatorname{Gal}\left(E^{\mathrm{ac}} / E\right)\right) .
$$

In this paper we focus on their restriction to $G\left(\mathbb{A}^{\infty}\right) \times W_{E_{v}}$, for $W_{E_{v}}$ the Weil group of the completion of $E$ at a prime $v$ dividing $p$.

2.2.2 Let $v$ be prime of $E$ dividing $p$. We write $E_{v}$ for the completion of $E$ at $v, \mathcal{O}_{E_{v}}$ for its ring of integers, and $k$ for it residue field, $\# k=q=p^{f}$. We choose an algebraic closure $k^{\mathrm{ac}}$ of $k$, and denote by $E_{0}$ the fraction field of $W\left(k^{\mathrm{ac}}\right)$, the ring of Witt vector of $k^{\text {ac }}$, and by $\sigma$ the $q$-th power of Frobenius on $E_{0}$. The unramified hypothesis in 2.1.2 implies that $v$ is unramified, i.e. $E_{v} \subset E_{0}$. We fix an algebraic closure $E_{v}^{\mathrm{ac}}$ of $E_{v}$, containing $E_{0}$, and an embedding $\bar{v}: E^{\mathrm{ac}} \rightarrow E_{v}^{\mathrm{ac}}$, extending the identity of $E$. These data allow us to identify the Weil group of $E_{v}$ with the decomposition group of $\bar{v}$ in $\operatorname{Gal}\left(E^{\mathrm{ac}} / E\right)$, i.e. $W_{E_{v}} \subset \operatorname{Gal}\left(E^{\mathrm{ac}} / E\right)$. For each $i \geq 0$, we regard the cohomology spaces $H_{c}^{i}\left(\operatorname{Sh}, \mathcal{L}_{\xi}\right)$ as $G\left(\mathbb{A}^{\infty}\right) \times W_{E_{v}}$-modules via restriction. They are admissible/continuous $G\left(\mathbb{A}^{\infty}\right) \times W_{E_{v}}$-modules which can be computed as

$$
H_{c}^{i}\left(\mathrm{Sh}, \mathcal{L}_{\xi}\right)=\lim _{K} H_{c}^{i}\left(\mathrm{Sh}_{K} \times_{E} E_{v}^{\mathrm{ac}}, \mathcal{L}_{\xi, K}\right)
$$

In the following, for each level $K$, we replace the $E$-scheme $\mathrm{Sh}_{K}$ by the $E_{v}$-scheme $\operatorname{Sh}_{K} \times_{\operatorname{Spec}(E)} \operatorname{Spec}\left(E_{v}\right)$, which with abuse of notations we still denote by $\mathrm{Sh}_{K}$.

2.2.3 Let $K$ be an open compact subgroup of $G\left(\mathbb{A}^{\infty}\right)$ as in Section 2.1.3, and consider the $\mathcal{O}_{E_{v}}$-scheme $S_{K} \times_{\operatorname{Spec}\left(\mathcal{O}_{E,(p)}\right)} \operatorname{Spec}\left(\mathcal{O}_{E_{v}}\right)$, which again with abuse of notations we still denote by $S_{K}$. We write $\bar{S}_{K}$ for its reduction in positive characteristic $p$, i.e. $\bar{S}_{K}=S_{K} \times_{\operatorname{Spec}\left(\mathcal{O}_{E_{v}}\right)} \operatorname{Spec}(k)$, and $R \Psi_{\eta}^{S}=R \Psi_{\eta}^{S_{K}}$ for its vanishing cycle functor. For all integers $p, q \geq 0$, we define

$$
\varliminf_{K} H_{c}^{p}\left(\bar{S}_{K} \times_{k} k^{\mathrm{ac}}, R^{q} \Psi_{\eta}^{S}\left(\mathcal{L}_{\xi}\right)\right)=H_{c}^{p}\left(\bar{S}, R^{q} \Psi_{\eta}^{S}\left(\mathcal{L}_{\xi}\right)\right) \text {. }
$$


It follows from the above constructions that these $l$-adic spaces have a natural structure of $G\left(\mathbb{A}^{\infty}\right)^{\dagger} \times W_{E_{v}}$-modules. In fact, they are admissible/continuous $G\left(\mathbb{A}^{\infty}\right)^{\dagger} \times W_{E_{v}}$-modules. We define

$$
H_{c}\left(\bar{S}, R \Psi_{\eta}^{S}\left(\mathcal{L}_{\xi}\right)\right)=\sum_{p, q}(-1)^{p+q} H_{c}^{p}\left(\bar{S}, R^{q} \Psi_{\eta}^{S}\left(\mathcal{L}_{\xi}\right)\right)
$$

The main goal of this paper is to study these virtual $G\left(\mathbb{A}^{\infty}\right)^{\dagger} \times W_{E_{v}}$-modules.

2.2.4 Let $K$ be of the form $K=K^{p} K_{p, 0}$. Then, the above vanishing cycle sheaves are easy to compute. More precisely, it follows from the construction of the integral models $S_{K}=S_{K^{p}}$ that in this case the lisse étale sheaf $\mathcal{L}_{\xi}$ over $\mathrm{Sh}_{K}$ extends canonically to a lisse étale sheaf over $S_{K^{p}}$ (which we also denote by $\mathcal{L}_{\xi}=\mathcal{L}_{\xi, K^{p}}$ ). Furthermore, given that the schemes $S_{K^{p}}$ are smooth, the associated vanishing cycle sheaves $R^{q} \Psi_{\eta}^{S}\left(\mathcal{L}_{\xi}\right)$ over $\bar{S}_{K^{p}} \times_{\operatorname{Spec}(k)} \operatorname{Spec}\left(k^{\text {ac }}\right)$ vanish for all $q \geq 1$, and for $q=0$ they are equal to the restriction of sheaf $\mathcal{L}_{\xi} / S_{K^{p}}$ to the geometric special fiber $\bar{S}_{K^{p}} \times_{\operatorname{Spec}(k)} \operatorname{Spec}\left(k^{\text {ac }}\right)$. In the following, we study the sheaves $R^{q} \Psi_{\eta}^{S_{K}}\left(\mathcal{L}_{\xi}\right)$, when the level $K$ is of the form $K=K^{p} K_{p, m}$, for an integer $m \geq 1$.

2.2.5 We remark that in the case of proper Shimura varieties, the theory of vanishing cycle sheaves ([4]) enable us to express the cohomology of the Shimura varieties in terms of the above cohomology spaces. More precisely, we deduce an equality of virtual admissible/continuous $G\left(\mathbb{A}^{\infty}\right)^{\dagger} \times W_{E_{v}}$-modules

$$
H\left(\mathrm{Sh}, \mathcal{L}_{\xi}\right)=H\left(\bar{S}, R \Psi_{\eta}^{S}\left(\mathcal{L}_{\xi}\right)\right),
$$

where the action of $G\left(\mathbb{A}^{\infty}\right)^{\dagger} \times W_{E_{v}}$ on the left hand side is obtained by restriction from the action of $G\left(\mathbb{A}^{\infty}\right) \times W_{E_{v}}$. Furthermore, in [13] (Section 8, p. 607), we showed that, under the assumption of properness, the action of $G\left(\mathbb{A}^{\infty}\right)^{\dagger} \times W_{E_{v}}$ on the right hand side canonically extends to the whole group $G\left(\mathbb{A}^{\infty}\right) \times W_{E_{v}}$, and that the above equality holds as an equality of $G\left(\mathbb{A}^{\infty}\right) \times W_{E_{v}}$-modules. To be precise, in loc. cit. we prove these results for $\xi=\mathbb{Q}_{l}^{\text {ac }}$ but the same arguments apply to any $\xi$. These results rely on a construction of integral correspondences which model the action of the whole $G\left(\mathbb{A}^{\infty}\right)$ on the Shimura varieties, as mentioned at the end of Section 2.1.3.

2.3 Newton stratification. We study the modules $H_{c}\left(\bar{S}, R \Psi_{\eta}^{S}\left(\mathcal{L}_{\xi}\right)\right)$ following the strategy carried out in [13], in the case of $\xi=\mathbb{Q}_{l}^{\text {ac }}$ the trivial representation. In this section, we recall the aspects of the general theory of local models for Shimura varieties of PEL type (following the work of Rapoport and Zink in [18]) and of the constructions in [13] which are relevant to extending the results to the case of $\xi$ any finite dimensional $l$-adic representation. We refer to [13] (Section 5, pp. 589-596) for details.

2.3.1 To the global Shimura datum $(G, h)$, we associate the local datum $\left(G_{\mathbb{Q}_{p}}, \mu_{\mathbb{Q}_{p}^{\text {ac }}}\right)$ where $G_{\mathbb{Q}_{p}}=G \otimes \mathbb{Q}_{\mathbb{Q}_{p}}$ and $\mu_{\mathbb{Q}_{p}^{\text {ac }}}$ denotes the conjugacy class of cocharacters of $G_{\mathbb{Q}_{p}}$ determined by $h$ and our choice of an embedding $\bar{v}: E^{\text {ac }} \rightarrow E_{v}^{\text {ac }}$, i.e. the class of cocharacters $\bar{v} \circ \mu_{h}$, for $\mu_{h}$ in $h$ (here, $\mathbb{Q}_{p}^{\text {ac }}=E_{v}^{\text {ac }}$ ). In [11], to a local datum $\left(G_{\mathbb{Q}_{p}}, \mu_{\mathbb{Q}_{p}}\right)$ Kottwitz associates a partially ordered finite set $B\left(G_{\mathbb{Q}_{p}}, \mu_{\mathbb{Q}_{p}}\right)$. For $G_{Q_{p}}$ of PEL type (which includes all cases coming from a global Shimura datum of PEL type $)$, the set $B\left(G_{\mathbb{Q}_{p}}, \mu_{\mathbb{Q}_{p}^{a c}}\right)$ is canonically identified with the set of isogeny classes of Barsotti-Tate groups over $k^{\text {ac }}$ with $\mu_{\mathbb{Q}_{p}^{\text {ac }}}$-compatible $G_{\mathbb{Q}_{p}}$-structures. In our cases, these include (and conjecturally are exactly) the Barsotti-Tate groups arising as the p-divisible parts of the abelian varieties over $k^{\mathbf{a c}}$ classified by the 
Shimura varieties associated with the corresponding global Shimura datum. Equivalently, $B\left(G_{\mathbb{Q}_{p}}, \mu_{\mathbb{Q}_{p}^{a c}}\right)$ is identified with a subset of the set of Newton polygons with end-points prescribed by $\mu_{\mathbb{Q}_{p}^{\text {ac }}}$. Under the latter identification, the partial order on $B\left(G_{\mathbb{Q}_{p}}, \mu_{\mathbb{Q}_{p}^{a c}}\right)$ corresponds to the partial order "lying on or below" among convex polygons ([17], Section 1, pp. 155-164).

2.3.2 Let $K$ be a sufficiently small open compact subgroup of $G\left(\mathbb{A}^{\infty}\right)$, and $\bar{S}_{K}$ the reduction modulo $p$ of the corresponding Shimura variety. For each geometric point $x$ of $\bar{S}_{K}$, we write $A_{x}$ for the corresponding abelian varieties, and $b_{x}$ for the isogeny class of the Barsotti-Tate group with additional structure $A_{x}\left[p^{\infty}\right]$. To each $b \in B\left(G_{\mathbb{Q}_{p}}, \mu_{\mathbb{Q}_{p}^{a}}\right)$, we associate the set

$$
\bar{S}_{K}(b)=\left\{x \in \bar{S}_{K} \mid b_{x}=b\right\} \text {. }
$$

The set $\bar{S}_{K}(b)$ is a locally closed subspace of $\bar{S}_{K}$, and has a natural structure of locally closed subscheme defined over $k$. Furthermore, as $b \in B\left(G_{\mathbb{Q}_{p}}, \mu_{\mathbb{Q}_{p}^{\text {ac }}}\right)$ varies, the subschemes $\bar{S}_{K}(b)$ form a stratification of $\bar{S}_{K}$ which is known as the Newton stratification. (The definition of the Newton stratification is due to Rapoport and Richartz in [17]. Their work generalizes to the context of Shimura varieties a classical result of Grothendieck, in [6]). We recall that it is not known in general whether the strata $\bar{S}_{K}(b)$ are non-empty for all $b \in B\left(G_{\mathbb{Q}_{p}}, \mu_{\mathbb{Q}_{p}^{a c}}\right)$. In the following, we only consider elements $b \in B\left(G_{\mathbb{Q}_{p}}, \mu_{\mathbb{Q}_{p}^{a c}}\right)$ which are associated with non-empty strata. We remark that this condition is independent of the level $K$. It is an easy observation that, for each $b \in B\left(G_{\mathbb{Q}_{p}}, \mu_{\mathbb{Q}_{p}}\right)$, as the level $K$ varies, the strata $\bar{S}_{K}(b)$ form a projective system stable under the action of $G\left(\mathbb{A}^{\infty}\right)^{\dagger}$. It follows that, for all $p, q \geq 0$, the $\mathbb{Q}_{l}^{a c}$-spaces

$$
H_{c}^{p}\left(\bar{S}(b), R^{q} \Psi_{\eta}^{S}\left(\mathcal{L}_{\xi}\right)\right)=\lim _{K} H_{c}^{p}\left(\bar{S}_{K}(b) \times_{k} k^{\mathrm{ac}}, R^{q} \Psi_{\eta}^{S}\left(\mathcal{L}_{\xi}\right)_{\mid \bar{S}_{K}(b)}\right)
$$

have a natural structure of admissible/continuous $G\left(\mathbb{A}^{\infty}\right)^{\dagger} \times W_{E_{v}}$-modules. For each $b \in B\left(G_{\mathbb{Q}_{p}}, \mu_{\mathbb{Q}_{p}^{a c}}\right)$, we define

$$
H_{c}\left(\bar{S}(b), R \Psi_{\eta}^{S}\left(\mathcal{L}_{\xi}\right)\right)=\sum_{p, q}(-1)^{p+q} H_{c}^{p}\left(\bar{S}(b), R^{q} \Psi_{\eta}^{S}\left(\mathcal{L}_{\xi}\right)\right)
$$

Then, there is an equality of virtual admissible/continuous $G\left(\mathbb{A}^{\infty}\right)^{\dagger} \times W_{E_{v}}$-modules

$$
H_{c}\left(\bar{S}, R \Psi_{\eta}^{S}\left(\mathcal{L}_{\xi}\right)\right)=\sum_{b \in B\left(G_{\mathrm{Q}_{p}}, \mu_{\mathrm{Q}_{p}^{\mathrm{ac}}}\right)} H_{c}\left(\bar{S}(b), R \Psi_{\eta}^{S}\left(\mathcal{L}_{\xi}\right)\right) .
$$

Furthermore, it follows from the remark in Section 2.2.5 that in the case of proper Shimura varieties the above equality holds as an equality of $G\left(\mathbb{A}^{\infty}\right) \times W_{E_{v}}$-modules.

2.4 Local models. Given the latter equality, we shift our focus to studying the modules $H_{c}\left(\bar{S}(b), R \Psi_{\eta}^{S}\left(\mathcal{L}_{\xi}\right)\right)$, for $b \in B\left(G_{\mathbb{Q}_{p}}, \mu_{\mathbb{Q}_{p}^{a c}}\right)$. Our strategy is to establish a connection between these spaces and the cohomology of the corresponding local models. In the following, we recall the main aspects of the theory of local models for Shimura varieties of PEL type. We refer to [18] for a complete discussion of this theory, and to [12] (Section 7.2, pp. 298-302, and Section 8.2, pp. 317-327) for details and proof of the results in Sections 2.4.2 and 2.4.3. 
2.4.1 Let $b \in B\left(G_{\mathbb{Q}_{p}}, \mu_{\mathbb{Q}_{p}^{a c}}\right)$. In [18], Rapoport and Zink associate to the data $\left(G_{\mathbb{Q}_{p}}, \mu_{\mathbb{Q}_{p}^{a c}}, b\right)$ a projective system of rigid analytic spaces $\mathcal{M}_{b, K_{p}}$ over $E_{0}$, indexed by the open compact subgroups $K_{p} \subset K_{p, 0}$ of $G\left(\mathbb{Q}_{p}\right)$, and naturally endowed with an action of $G\left(\mathbb{Q}_{p}\right)$ and a $\sigma$-semi-linear automorphism. For $K_{p}=K_{p, 0}$, the rigid analytic space $\mathcal{M}_{b, K_{p, o}}$ is defined as the generic fiber of the formal $W\left(k^{\text {ac }}\right)$-scheme representing the following set-valued functor $M_{b}$ on the category of formal $W\left(k^{\mathrm{ac}}\right)$ schemes. We first define $M_{b}=M_{b, \mathbb{X}}$ as depending on the choice of a Barsotti-Tate group with additional structure $\mathbb{X} / k^{\text {ac }}$ in the isogeny class determined by $b$. We later observe that the resulting functor is up to isomorphism independent of such a choice. We remark that it suffices to define $M_{b, \mathbb{X}}(S)$ for any $W\left(k^{\text {ac }}\right)$-scheme $S$ where $p$ is locally nilpotent. Let $M_{b, \mathbb{X}}(S)$ be the set of isomorphism classes of pairs $(H, \beta)$ where $H$ is a Barsotti-Tate group with additional structures defined over $S$, and $\beta: \mathbb{X}_{\bar{S}} \rightarrow H_{\bar{S}}$ is a quasi-isogeny defined over the subscheme $\bar{S}=Z(p)$ of $S$. (Here, $Z(p)$ denotes the subscheme defined by the equation $p=0$, regarded as a $k^{\text {ac }}$-scheme.) The functor $M_{b, \mathbb{X}}$ is represented by a formal $W\left(k^{\mathrm{ac}}\right)$-scheme $\mathcal{M}_{b, \mathrm{X}}$, which is formally smooth and formally locally of finite type. The formal scheme $\mathcal{M}_{b, \mathbb{X}}$ is naturally endowed with an action of the group of quasi-self-isogenies of $\mathbb{X}$, by right translations. We denote the group of quasi-self-isogenies of $\mathbb{X}$ by $J_{b}\left(\mathbb{Q}_{p}\right)$, since it may be canonically identified with the $\mathbb{Q}_{p}$-points of an algebraic group $J_{b} / \mathbb{Q}_{p}$, depending only on $b \in B\left(G_{\mathbb{Q}_{p}}, \mu_{\mathbb{Q}_{p}^{\text {ac }}}\right)$. Furthermore, the scheme $\mathcal{M}_{b, \mathbb{X}}$ is also endowed with a $\sigma$-semi-linear automorphism, which is defined by left translation by the inverse of the $q$-th power of the Frobenius of $\mathbb{X}$, and which commutes with the previous action of $J_{b}\left(\mathbb{Q}_{p}\right)$. In the following, we refer to this automorphism as an (unramified) action of $W_{E_{v}}$. Finally, for each pair of non-negative integers $n, d \geq 0$, we define the truncated Rapoport-Zink space $\mathcal{M}_{b, \mathrm{X}}^{n, d}$ to be the formal subscheme of $\mathcal{M}_{b, \mathrm{X}}$ classifying pairs $(H, \beta)$ such that the quasi-isogenies $p^{n} \beta$ and $p^{d-n} \beta^{-1}$ are isogenies. For all $n, d$, the natural inclusions $\mathcal{M}_{b, \mathbb{X}}^{n, d} \rightarrow \mathcal{M}_{b, \mathbb{X}}$ are closed embeddings and, as $n, d$ vary, they define a direct system. Let $\mathcal{M}_{b, K_{p, 0}}$ denote the generic fiber of the formal $W\left(k^{\text {ac }}\right)$-scheme $\mathcal{M}_{b, \mathbb{X}}$, regarded as a rigid analytic $E_{0}$-space. For each $K_{p} \subset K_{p, 0}$, we define $\mathcal{M}_{b, K_{p}}$ as the space over $\mathcal{M}_{b, K_{p, 0}}$ parameterizing $K_{p}$-level structures on the universal Barsotti-Tate group $H / \mathcal{M}_{b, K_{p, 0}}$ (here, by a $K_{p}$-level structure on $H$ we mean the $K_{p}$-orbit of a trivialization of the Tate module of $H$ ). For each $K_{p} \subset K_{p, 0}$, the rigid analytic space $\mathcal{M}_{b, K_{p}}$ is a finite étale Galois cover of $\mathcal{M}_{b, K_{p, o}}$. In particular, it is a smooth rigid analytic space over $E_{0}$, of dimension $D_{b}=\operatorname{dim} \mathcal{M}_{b, K_{p, 0}}$.

2.4.2 As $K_{p}$ varies, the spaces $\mathcal{M}_{b, K_{p}}$ form a projective system naturally endowed with an action of $G\left(\mathbb{Q}_{p}\right) \times J_{b}\left(\mathbb{Q}_{p}\right) \times W_{E_{v}}$. For each $i \geq 0$, we define

$$
H_{c}^{i}\left(\mathcal{M}_{b}, \mathbb{Q}_{l}^{\mathrm{ac}}\right)=\lim _{\longrightarrow} K_{p} H_{c}^{i}\left(\mathcal{M}_{b}, K_{p} \times E_{0} E_{v}^{\mathrm{ac}}, \mathbb{Q}_{l}^{\mathrm{ac}}\right) .
$$

Then, they are smooth/continuous $G\left(\mathbb{Q}_{p}\right) \times J_{b}\left(\mathbb{Q}_{p}\right) \times W_{E_{v}}$-modules, but it is not known whether they are also admissible/continuous. On the other hand, to each admissible representation $\rho$ of $J_{b}\left(\mathbb{Q}_{p}\right)$, we may associated the $G\left(\mathbb{Q}_{p}\right) \times W_{E_{v}}$-modules $\operatorname{Ext}_{J_{b}\left(\mathbb{Q}_{p}\right)}^{p}\left(H_{c}^{q}\left(\mathcal{M}_{b}, \mathbb{Q}_{l}^{a c}\right), \rho\right)$. These modules are admissible/continuous and vanish for almost all $p, q \geq 0$. For each $b \in B\left(G_{\mathbb{Q}_{p}}, \mu_{\mathbb{Q}_{p}}\right)$, we define a functor

$$
\mathcal{E}_{b}: \operatorname{Groth}\left(J_{b}\left(\mathbb{Q}_{p}\right)\right) \rightarrow \operatorname{Groth}\left(G\left(\mathbb{Q}_{p}\right) \times W_{E_{v}}\right)
$$


which to an admissible virtual representation $\rho$ of $J\left(\mathbb{Q}_{p}\right)$ associates

$$
\mathcal{E}_{b}(\rho)=\sum_{p, q}(-1)^{p+q} \operatorname{Ext}_{J_{b}\left(\mathbb{Q}_{p}\right)}^{p}\left(H_{c}^{q}\left(\mathcal{M}_{b}, \mathbb{Q}_{l}^{\mathrm{ac}}\right), \rho\right)\left(-D_{b}\right)
$$

In the following we also denote by $\mathcal{E}_{b}$ the functor from $\operatorname{Groth}\left(J_{b}\left(\mathbb{Q}_{p}\right) \times G\left(\mathbb{A}^{\infty, p}\right)\right)$ to $\operatorname{Groth}\left(G\left(\mathbb{A}^{\infty}\right) \times W_{E_{v}}\right)$ which is obtain by extending the above functor by the identity on $\operatorname{Groth}\left(G\left(\mathbb{A}^{\infty, p}\right)\right)$.

2.4.3 It follows from Berkovich's theory of vanishing cycles of formal schemes ([3], Corollary 2.5, p. 373), together with the fact that all the irreducible components of the reduced scheme associated with $\mathcal{M}_{b, \mathbf{X}}$ are projective $k^{\text {ac }}$-schemes ([18], Proposition 2.32, p. 63), that the above functor can also be computed in terms of the cohomology groups of the reduced closed fiber of $\mathcal{M}_{b, \mathbb{x}}$ with coefficients in the appropriate vanishing cycle sheaves. Indeed, let us restrict our attention to levels $K_{p}$ of the form $K_{p}=K_{p, m}, m \geq 0$. Then, similarly to the case of the integral models of Shimura varieties of PEL type, we can apply Katz and Mazur's notion of full set of sections for finite flat group schemes to construct integral models for the Rapoport-Zink spaces. More precisely, for each $K_{p}=K_{p, m}, m \geq 0$, there exists a formal $W\left(k^{\text {ac }}\right)$-scheme $\mathcal{M}_{b, \mathbb{X}, K_{p}}$ satisfying the following properties. It is finite over $\mathcal{M}_{b, \mathbb{X}}=\mathcal{M}_{b, \mathbb{X}, K_{p}, 0}$, and its generic fiber is canonically isomorphic to $\mathcal{M}_{b, K_{p}}$. Furthermore, as $K_{p}$ varies, the formal schemes $\mathcal{M}_{b, \mathrm{X}, K_{p}}$ form a projective system endowed with an action of $J_{b}\left(\mathbb{Q}_{p}\right) \times G\left(\mathbb{Q}_{p}\right)^{\dagger} \times W_{E_{v}}$, which is compatible under the above isomorphisms with the action of $J_{b}\left(\mathbb{Q}_{p}\right) \times G\left(\mathbb{Q}_{p}\right) \times W_{E_{v}}$ on the rigid analytic Rapoport-Zink spaces $\mathcal{M}_{b, K_{p}}$. For each $K_{p}=K_{p, m}$, we write $\overline{\mathcal{M}}_{b, K^{p}}=\overline{\mathcal{M}}_{b, \mathrm{X}, K_{p}}$ for the reduced scheme associated with $\mathcal{M}_{b, \mathbf{X}, K^{p}}$, regarded as a $k^{\text {ac }}$-scheme, and denote by $R \Psi_{\eta}^{\mathcal{M}}=R \Psi_{\eta}^{\mathcal{M}_{K_{p}}}$ its vanishing cycle functor. Then, for each $p, q \geq 0$, we define the $\mathbb{Q}_{l}^{a c}$-space

$$
H_{c}^{p}\left(\overline{\mathcal{M}}_{b}, R^{q} \Psi_{\eta}^{\mathcal{M}}\left(\mathbb{Q}_{l}^{\mathrm{ac}}\right)\right)=\underline{\lim }_{\longrightarrow} K_{p} H_{c}^{p}\left(\overline{\mathcal{M}}_{b, K_{\mathrm{p}}}, R^{q} \Psi_{\eta}^{\mathcal{M}_{K_{p}}}\left(\mathbb{Q}_{l}^{\mathrm{ac}}\right)\right) .
$$

For all $p, q \geq 0$, these are smooth/continuous $G\left(\mathbb{Q}_{p}\right) \times J_{b}\left(\mathbb{Q}_{p}\right) \times W_{E_{v}}$-modules. Moreover, for each admissible representation $\rho$ of $J_{b}\left(\mathbb{Q}_{p}\right)$, the $G\left(\mathbb{Q}_{p}\right)^{\dagger} \times W_{E_{v}}$-modules $\operatorname{Tor}_{J_{b}\left(\mathbb{Q}_{p}\right)}^{i}\left(H_{c}^{p}\left(\overline{\mathcal{M}}_{b}, R^{q} \Psi_{\eta}^{\mathcal{M}}\left(\mathbb{Q}_{l}^{a c}\right)\right), \rho\right)$ are admissible/continuous and vanish for almost all $i, p, q \geq 0$. Similarly to the case of integral models for Shimura varieties, by considering a class of integral models for the Rapoport-Zink spaces larger than that introduced here, it is possible to canonically extend the smooth/continuous (resp. admissible /continuous) action of $G\left(\mathbb{Q}_{p}\right)^{\dagger} \times W_{E_{v}}$ on the above $\mathbb{Q}_{l}^{\text {ac }}$-spaces to a smooth/continuous (resp. admissible/continuous) action of $G\left(\mathbb{Q}_{p}\right) \times W_{E_{v}}$. Furthermore, for each $\rho$, there is an equality of virtual $G\left(\mathbb{Q}_{p}\right) \times W_{E_{v}}$-modules

$$
\mathcal{E}_{b}(\rho)=\sum_{i, p, q}(-1)^{i+p+q} \operatorname{Tor}_{J_{b}\left(\mathbb{Q}_{p}\right)}^{i}\left(H_{c}^{p}\left(\overline{\mathcal{M}}_{b}, R^{q} \Psi_{\eta}^{\mathcal{M}}\left(\mathbb{Q}_{l}^{a c}\right)\right), \rho\right)
$$

2.5 Igusa varieties. In order to establish a connection between the cohomology of the Shimura varieties and that of their local models, we introduce a new kind of moduli spaces for abelian varieties in positive characteristic, the Igusa varieties. Igusa curves were first introduced by Igusa in the theory of modular curves ([8], or [9], chapters 12 and 13 , pp. 344-450), and later generalized in the context of some simple Shimura varieties by Harris and Taylor ([7]). Their more general definition in the context of Shimura varieties of PEL type relies on work of Oort on foliations in moduli spaces of abelian varieties in positive characteristic ([15]), and on results 
of Zink ([19]), and of Oort and Zink ([16]) on the existence of the slope filtration for p-divisible groups. We refer to [13] (Section 4, pp. 584-589) for details.

2.5.1 Let $b \in B\left(G_{\mathbb{Q}_{p}}, \mu_{\mathbb{Q}_{p}^{a c}}\right)$, and fix a level $K^{p} \subset G\left(\mathbb{A}^{\infty, p}\right)$. We assume $\bar{S}_{K^{p}}(b)$ is not empty, and choose $x$ a closed geometric point of $\bar{S}_{K^{p}}(b)$, defined over $k^{\text {ac }}$. We write $\mathbb{X}=A_{x}\left[p^{\infty}\right]$. The Barsotti-Tate group $\mathbb{X} / k^{\text {ac }}$ admits a unique slope decomposition, $\mathbb{X}=\oplus_{1}^{t} \mathbb{X}^{i}$, into isoclinic Barsotti-Tate subgroups $\mathbb{X}^{i}$, each endowed with the appropriate inherited additional structures (i.e. an action of $\mathcal{O}_{B_{\mathbf{Q}_{p}}}$, and isomorphisms $\ell^{i}: \mathbb{X}^{i} \cong\left(\mathbb{X}^{t+1-i}\right)^{\vee}$ induced by the quasi-polarization $\ell$ of $\left.\mathbb{X}\right)$. As before, we write $J_{b}\left(\mathbb{Q}_{p}\right)$ for the group of quasi-self-isogenies of $\mathbb{X}$. We recall that the associated algebraic group $J_{b} / \mathbb{Q}_{p}$ is an inner form of the Levi subgroup of $G_{\mathbb{Q}_{p}}$ associated with the slope decomposition of the isocrystals of $\mathbb{X}$.

2.5.2 We say that a Barsotti-Tate group with additional structures $Y$ defined over field $k^{\prime} \supset k^{\text {ac }}$ is geometrically isomorphic to $\mathbb{X}$ (we write $Y \cong_{g} \mathbb{X}$ ) if there exists a field extension $k^{\prime \prime} \supset k^{\prime}$ over which the two become isomorphic. We define $C=C_{x}=C_{\mathbb{X}}$, the central leaf through $x$ (or equivalently, associated to $\mathbb{X}$ ), as

$$
C_{x}=\left\{y \in \bar{S}_{K^{p}}(b) \mid A_{y}\left[p^{\infty}\right] \cong \cong_{g} \mathbb{X}\right\} .
$$

Then, $C_{x}$ is a closed subspace of $\bar{S}_{K^{p}}(b)$ defined over $k^{\text {ac }}$, which is a smooth scheme when endowed with the reduced subscheme structure. We regard $C$ as a smooth closed $k^{\text {ac }}$-subscheme of $\bar{S}_{K^{p}}(b)$. For each $K^{p}$, we also write $C=C_{K^{p}} \subset \bar{S}_{K^{p}}(b)$. Then, as $K^{p}$ varies, the schemes $C_{K^{p}}$ naturally form a projective system endowed with an action of $G\left(\mathbb{A}^{\infty, p}\right) \subset G\left(\mathbb{A}^{\infty}\right)^{\dagger}$.

2.5.3 Let $C=C_{\mathbb{X}, K^{p}}$. Without loss of generality, we assume $\mathbb{X}$ is completely slope divisible. We write $H / C$ for the p-divisible part of the restriction of the universal abelian scheme $A / \bar{S}_{K^{p}}(b)$ to $C$. Then, it follows from the work of Zink ([19]), and of Oort and Zink ([16]), that the Barsotti-Tate group $H / C$ is also completely slope divisible. More precisely, $H$ admits a canonical slope filtration $0=H_{0} \subset H_{1} \subset \cdots H_{t}=H$, into Barsotti-Tate subgroups with slope divisible Barsotti-Tate subquotients $H^{i}=H_{i} / H_{i-1}$. Each $H^{i} / C$ is a Barsotti-Tate group endowed with appropriate additional structures inherited by those of $H$. Moreover, it follows from the definition of $C$ that, for each point $y \in C$, the Barsotti-Tate groups with additional structures $H_{y}^{i}$ are geometrically isomorphic to $\mathbb{X}^{i}$, for all $i$. We write $H^{s p}=\oplus_{1}^{t} H^{i}$, also regarded as a Barsotti-Tate group with additional structure (its additional structures controlled by the Levi subgroup of $G_{\mathbb{Q}_{p}}$ which is an inner form of $J_{b}$ ). We observe that, for each $y \in C$, the Barsotti-Tate group $H_{y}^{s p}$ is geometrically isomorphic (via a canonical isomorphism) to $H_{y}$. (This follows from the fact that the slope filtration of a Barsotti-Tate group canonically splits over an algebraically closed field.)

2.5.4 For each $m \geq 1$, we define the Igusa variety of level $m$ as the space $\operatorname{Ig}_{m}=\operatorname{Ig}_{\mathbb{X}, K^{p}, m}$ over $C_{\mathbb{X}, K^{p}}$ parameterizing isomorphisms of truncated BarsottiTate groups with additional structures $j_{m}: \mathbb{X}\left[p^{m}\right]_{C} \cong H^{s p}\left[p^{m}\right]$. (Here, by an isomorphism of truncated Barsotti-Tate group with additional structures, we mean an isomorphism of the underlying finite flat group schemes, which commutes with the additional structures and which extends étale locally to any truncation of higher depth $m^{\prime} \geq m$.) It follows from the definition that, for each $m \geq 1$, the Igusa variety $\mathrm{Ig}_{m}$ is finite étale and Galois over $C$. Thus, in particular, it is a smooth scheme over $k^{\mathrm{ac}} . \mathrm{Ig}_{m}$ is naturally endowed with an action of the group of automorphism of $\mathbb{X}$. Further more, as $K^{p}$ and $m$ vary, the schemes $\operatorname{Ig}_{K^{p}, m}$ naturally form a 
projective system endowed with commuting actions of $G\left(\mathbb{A}^{\infty, p}\right) \subset G\left(\mathbb{A}^{\infty}\right)^{\dagger}$ and the group of automorphism of $\mathbb{X}$. If we regard the group of automorphism of $\mathbb{X}$ as an open compact subgroup of $J_{b}\left(\mathbb{Q}_{p}\right)$, then its action on the projective system of Igusa varieties naturally extends to a larger subgroup which we denote by $J_{b}\left(\mathbb{Q}_{p}\right)^{\dagger}([13]$, Section 4, Lemma 5, pp. 586-587).

2.5.5 Let $\xi$ be a finite dimensional $l$-adic representation of $G$ and $\mathcal{L}_{\xi}$ the associated lisse étale sheaf over the models $S_{K^{p}}$ as in Section 2.2.4. For all $K^{p}, m$ as above, we write $\mathcal{L}_{\xi \mid \operatorname{Ig}_{\mathbf{X}, K^{p}, m}}$ for the pullback of the sheaf $\mathcal{L}_{\xi} / S_{K^{p}}$ under the natural morphism $\operatorname{Ig}_{\mathbb{X}, K^{p}, m} \rightarrow C_{\mathbb{X}, K^{p}} \subset \bar{S}_{K^{p}} \subset S_{K^{p}}$. For each $i \geq 0$, we define

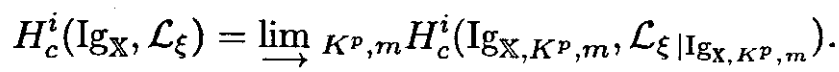

These $\mathbb{Q}_{l}^{\text {ac }}$-vector spaces have a natural structure of $G\left(\mathbb{A}^{\infty, p}\right) \times J_{b}\left(\mathbb{Q}_{p}\right)^{\dagger}$-modules, which canonically extends to a structure of $G\left(\mathbb{A}^{\infty, p}\right) \times J_{b}\left(\mathbb{Q}_{p}\right)$-modules. Further more, as $G\left(\mathbb{A}^{\infty, p}\right) \times J_{b}\left(\mathbb{Q}_{p}\right)$-modules they are admissible. We define

$$
H_{c}\left(\operatorname{Ig}_{\mathbb{X}}, \mathcal{L}_{\xi}\right)=\sum_{i}(-1)^{i} H_{c}^{i}\left(\operatorname{Ig}_{\mathbb{X}}, \mathcal{L}_{\xi}\right) \in \operatorname{Groth}\left(G\left(\mathbb{A}^{\infty, p}\right) \times J_{b}\left(\mathbb{Q}_{p}\right)\right)
$$

We remark that the above modules depend only on the isogeny class of $\mathbb{X}$. In the following, given $b \in B\left(G_{\mathbb{Q}_{p}}, \mu_{\mathbb{Q}_{p}^{a c}}\right)$, we sometimes write $H_{c}\left(\operatorname{Ig}_{b}, \mathcal{L}_{\xi}\right)=H_{c}\left(\operatorname{Ig}_{\mathbb{X}}, \mathcal{L}_{\xi}\right)$, for any choice of a Barsotti-Tate group $\mathbb{X}$ in the isogeny class determined by $b$.

\section{Statement of the main results}

We are finally read to state the main theorem of this paper, as well as the intermediate results necessary to adapt to this more general context the strategy of [12] and [13].

Theorem 3.1 Maintaining the above notations. Let $\xi$ be a representation of $G$ on a finite dimensional $\mathbb{Q}_{l}^{\text {ac }}$-vector space. For each $b \in B\left(G_{\mathbb{Q}_{p}}, \mu_{\mathbb{Q}_{p}^{a c}}\right)$, there is an equality of virtual $G\left(\mathbb{A}^{\infty}\right)^{\dagger} \times W_{E_{v}}$-modules

$$
H_{c}\left(\bar{S}(b), R \Psi_{\eta}^{S}\left(\mathcal{L}_{\xi}\right)\right)=\mathcal{E}_{b}\left(H_{c}\left(I_{b}, \mathcal{L}_{\xi}\right)\right)
$$

where $\mathcal{E}_{b}$ is the functor associated with the local models of the Shimura varieties attached to $b$. In the case of proper Shimura varieties, the above equalities can be extended to the equalities of $G\left(\mathbb{A}^{\infty}\right) \times W_{E_{u}}$-modules, for all $b \in B\left(G_{\mathbb{Q}_{p}}, \mu_{\mathbb{Q}_{p}^{a c}}\right)$. In particular, we deduce an equality of $G\left(\mathbb{A}^{\infty}\right) \times W_{E_{v}}$-modules

$$
H\left(S h, \mathcal{L}_{\xi}\right)=\sum_{b \in B\left(G_{Q_{p}, \mu_{\mathrm{Q}_{p}} \mathrm{ac}}\right)} \mathcal{E}_{b}\left(H\left(I g_{b}, \mathcal{L}_{\xi}\right)\right)
$$

For $\xi=\mathbb{Q}_{l}^{\text {ac }}$ the trivial representation, the above theorem was proven in [13] (Theorem 22, p. 607). While the above formula was previously only established for cohomology with trivial coefficients, many of the constructions and intermediate results in [12] and [13] were carried out in larger generality. In the following, we recall those constructions and point out the results about the local geometry of the Shimura varieties and their vanishing cycle sheaves which underlie the formula. 
3.1 Strategy. We fix $K^{p}$ a sufficiently small open compact subgroup of $G\left(\mathbb{A}^{\infty, p}\right)$. Let $b \in B\left(G_{\mathbb{Q}_{p}}, \mu_{\mathbb{Q}_{p}^{\text {ac }}}\right)$ and assume $\bar{S}(b)=\bar{S}_{K^{p}}(b)$ is not empty. We choose $\mathbb{X}$ a Barsotti-Tate group with additional structures associated with a closed geometric point of $\bar{S}(b)$, and write $\overline{\mathcal{M}}=\overline{\mathcal{M}}_{b, \mathrm{X}}$ (resp. $\overline{\mathcal{M}}^{n, d}=\overline{\mathcal{M}}_{b, \mathrm{X}}^{n, d}, n, d \geq 0$ ) for the reduced closed fiber of the local model $\mathcal{M}_{b, \mathrm{X}}\left(\right.$ resp. $\left.\mathcal{M}_{b, \mathrm{X}}^{n, d}\right)$. For each $m \geq 0$, we also write $\operatorname{Ig}_{m}=\operatorname{Ig}_{\mathbb{X}, K^{p}, m}$ for the Igusa variety of level $m$, associated with our choice of $b, \mathbb{X}$, and $K^{p}$. For all $m, n, d \geq 0$, we define

$$
q_{1}: \operatorname{Ig}_{m} \times \overline{\mathcal{M}}^{n, d} \rightarrow \operatorname{Ig}_{0} \text { and } q_{2}: \operatorname{Ig}_{m} \times \overline{\mathcal{M}}^{n, d} \rightarrow \overline{\mathcal{M}}
$$

respectively as the first projection of the product composed with the cover map $\mathrm{Ig}_{m} \rightarrow \mathrm{Ig}_{0}$, and the second projection of the product composed with the natural inclusion $\overline{\mathcal{M}}^{\text {nd }} \hookrightarrow \overline{\mathcal{M}}$. Finally, for any level $K$ of the form $K=K^{p} K_{p}$, with $K_{p}=K_{p, m} \subset K_{p, 0}$, we write $K_{0}=K^{p} K_{p, 0}$, and denote by $f_{K_{p}}: \mathrm{Sh}_{K} \rightarrow \mathrm{Sh}_{K_{0}}$ and $g_{K_{p}}: \mathcal{M}_{b, K_{p}} \rightarrow \mathcal{M}_{b, K_{p, 0}}$ the cover maps between Shimura varieties and RapoportZink spaces corresponding respectively to the inclusions $K \subset K_{0}$ and $K_{p} \subset K_{p, 0}$.

3.1.1 In [13] (Proposition 9, p.592) we construct a system of finite $k^{\text {ac }}$-morphisms

$$
\pi_{(N, m, n, d)}: \operatorname{Ig}_{m} \times \overline{\mathcal{M}}^{n, d} \rightarrow \bar{S}(b)_{k^{\mathrm{ac}}}=\bar{S}(b) \times_{k} k^{\mathrm{ac}},
$$

indexed by quadruples of non-negative integers $(N, m, n, d)$ satisfying certain conditions (namely, we require that $m, N$ are sufficiently large with respect to $d$ ). As $m, n, d$ vary, the morphisms $\pi_{(N, m, n, d)}$ form a system compatible under the natural projections among Igusa varieties and inclusion of truncated Rapoport-Zink spaces, and as $N$ varies, they are compatible up to powers of the relative Frobenius $F$ of $\bar{S}(b) / k$. Moreover, as a system, they are $J_{b}\left(\mathbb{Q}_{p}\right)^{\dagger}$-equivariant, for the natural action of $J_{b}\left(\mathbb{Q}_{p}\right)^{\dagger} \subset J_{b}\left(\mathbb{Q}_{p}\right)$ on the system of products $\operatorname{Ig}_{m} \times \overline{\mathcal{M}}^{n, d}$, and the trivial action on $\bar{S}(b)$. In the following, with abuse of notations we sometime write $\pi_{N}=\pi_{(N, m, n, d)}$. (We postpone recalling their definition to Section 5, proof of proposition 5.2.) The special case $\xi=\mathbb{Q}_{l}^{\text {ac }}$ of Theorem 3.1 is proved in [13] by combining together the following results.

Theorem 1 [12], Theorem 5.11, p. 287] Let $\mathcal{L}$ be an abelian torsion étale sheaf over $\bar{S}(b)_{k^{\text {ac }}}$, with torsion orders prime to $p$ and endowed with an action of $W_{E_{v}}$. There exists a $W_{E_{v}}$-equivariant spectral sequence

$$
E_{2}^{p, q}=H_{q}\left(J_{b}\left(\mathbb{Q}_{p}\right), \underline{\lim }_{m, n, d} H_{c}\left(I_{m} \times \overline{\mathcal{M}}^{n, d}, \pi_{N}^{*} \mathcal{L}\right)\right) \Rightarrow H_{c}^{p+q}\left(\bar{S}(b) \times_{k} k^{a c}, \mathcal{L}\right) .
$$

Theorem 2 [12], Theorem 5.13, p. 281] Let $r \geq 1$, and $\mathcal{L} / \bar{S}(b)_{k^{a c}}, \mathcal{G} / \overline{\mathcal{M}}$ be two étale sheaves of $\mathbb{Z} / l^{r} \mathbb{Z}$-modules, which are endowed with an action of $W_{E_{v}}$. We assume that there exists a system of $W_{E_{v}}$-equivariant isomorphisms, indexed by the quadruples $(N, m, n, d)$,

$$
\pi_{N}^{*} \mathcal{L} \cong q_{2}^{*} \mathcal{G},
$$

which are compatible under the morphisms induced by the projections among the Igusa varieties, the inclusion of truncated Rapoport-Zink spaces and the action of $J_{b}\left(\mathbb{Q}_{p}\right)^{\dagger}$. Then, there exists a $W_{E_{v}}$-equivariant spectral sequence

$$
E^{p, q}=\oplus_{t+s=q} \operatorname{Tor}_{J_{b}\left(\mathbb{Q}_{p}\right)}^{p}\left(H_{c}^{s}\left(\overline{\mathcal{M}}, \mathcal{G}_{2}\right), H_{c}^{t}\left(I g, \mathbb{Z} / l^{r} \mathbb{Z}\right)\right) \Rightarrow H_{c}^{p+q}\left(\bar{S}(b) \times_{k} k^{a c}, \mathcal{L}\right) .
$$


Theorem 3 [13], Proposition 21, p. 605] Let $K=K^{p} K_{p}$ be a level as in Section 2.1.3, $b \in B\left(G_{\mathbb{Q}_{p}}, \mu_{\mathbb{Q}_{p}^{a c}}\right)$. For each $r \geq 1$, there exists a system of $W_{E_{v}}-$ quasi-isomorphism of complexes, indexed by the quadruples $(N, m, n, d)$,

$$
\pi_{N}^{*}\left(R \Psi_{\eta}^{S} R f_{K_{p} *}\left(\mathbb{Z} / l^{r} \mathbb{Z}\right)_{\mid \bar{S}(b)_{k^{a c}}}\right) \cong q_{2}^{*}\left(R \Psi_{\eta}^{\mathcal{M}} R g_{K_{p} *}\left(\mathbb{Z} / l^{r} \mathbb{Z}\right)\right)
$$

which are compatible under the morphisms induced by the projections among the Igusa varieties, the inclusion of truncated Rapoport-Zink spaces and the action of $J_{b}\left(\mathbb{Q}_{p}\right)^{\dagger}$. Furthermore, they are also compatible with the action of $G\left(\mathbb{Q}_{p}\right)^{\dagger}$, as the level $K$ varies.

3.1.2 We state the appropriate analogues of Theorems 2 and 3 in the context of this paper.

Theorem 3.2 Let $r \geq 1$, and $\mathcal{L} / \bar{S}(b)_{k^{a c}}, \mathcal{G}_{1} / I g_{0}$ and $\mathcal{G}_{2} / \overline{\mathcal{M}}$ be some étale sheaves of $\mathbb{Z} / l^{r} \mathbb{Z}$-modules, which are endowed with an action of $W_{E_{v}}$. We assume that there exists a system of $W_{E_{v}}$-equivariant isomorphisms, indexed by the quadruples $(N, m, n, d)$,

$$
\pi_{N}^{*} \mathcal{L} \cong q_{1}^{*} \mathcal{G}_{1} \otimes q_{2}^{*} \mathcal{G}_{2},
$$

which are compatible under the morphisms induced by the projections among the Igusa varieties, the inclusion of truncated Rapoport-Zink spaces and the action of $J_{b}\left(\mathbb{Q}_{p}\right)^{\dagger}$. Then, there exists a $W_{E_{v}}$-equivariant spectral sequence

$$
E^{p, q}=\oplus_{t+s=q} \operatorname{Tor}_{J_{b}\left(\mathbb{Q}_{p}\right)}^{p}\left(H_{c}^{s}\left(\overline{\mathcal{M}}, \mathcal{G}_{2}\right), H_{c}^{t}\left(I g, \mathcal{G}_{1}\right)\right) \Rightarrow H_{c}^{p+q}\left(\bar{S}(b) \times_{k} k^{\mathrm{ac}}, \mathcal{L}\right) .
$$

Theorem 3.3 Let $\xi$ be a representation of $G$ on a finite dimensional $\mathbb{Q}_{l}^{a c_{-}}$ vector space, $b \in B\left(G_{\mathbb{Q}_{p}}, \mu_{\mathbb{Q}_{p}^{a c}}\right)$, and $K=K^{p} K_{p}$ a level as in Section 2.1.3. There exists a system of $W_{E_{v}}-q u a s i-i s o m o r p h i s m$ of complexes, indexed by the quadruples $(N, m, n, d)$,

$$
\pi_{N}^{*}\left(R \Psi_{\eta}^{S} R f_{K_{p} *}\left(\mathcal{L}_{\xi}\right)_{\mid \bar{S}(b)_{k^{a c}}}\right) \cong q_{1}^{*}\left(\mathcal{L}_{\xi \mid I g_{0}}\right) \otimes q_{2}^{*}\left(R \Psi_{\eta}^{\mathcal{M}} R g_{K_{p} *}\left(\mathbb{Q}_{l}^{a c}\right)\right),
$$

which are compatible under the morphisms induced by the projections among the Igusa varieties, the inclusion of truncated Rapoport-Zink spaces and the action of $J_{b}\left(\mathbb{Q}_{p}\right)^{\dagger}$. Furthermore, they are also compatible with the action of $G\left(\mathbb{Q}_{p}\right)^{\dagger}$, as the level $K$ varies.

Proof of Theorem 3.1 In view of the results discussed in Section 2, Theorem 3.1 is an immediate consequence of Theorems 3.2 and 3.3. In particular, in the case of proper Shimura varieties, following the discussions in Sections 2.2.5 and 2.4.3, we can deduce the second statement from Theorems 3.2 and 3.3, as long as we include in Theorem 3.3 the case of the vanishing cycle sheaves associated with a larger class of integral models.

Proof of Theorem 3.2 The techniques used in [12] to prove Theorem 2 apply directly to this more general case. (Indeed, Theorem 2 is a special case of Theorem 3.2 , namely the case of $\mathcal{G}_{1}=\mathbb{Q}_{l}^{\text {ac }}$.) More precisely, after applying the Künneth formula for étale cohomology to the products of Igusa varieties and Rapoport-Zink spaces, the statement follows from Theorem 1.

The next two sections are dedicated to proving Theorem 3.3. 


\section{Reduction to a special case}

In the previous section, we stated our results for $\xi$ a finite dimensional $l$-adic representation of $G$. It is an easy observation that in fact it is sufficient to consider the cases when $\xi$ is irreducible, since the general case would then follow from those. In this section, we explain how the special case of $\xi$ equal to the exterior algebra of sums of copies of the dual of the representation $V$ of $G$ implies all the cases of $\xi$ irreducible and thus the general statement. Further more, we explain how these cases are related to the study of the local geometry of the multi-self products of the universal abelian scheme over the Shimura varieties.

4.1 Preliminaries. Let $V_{0}$ denote the dual of the representation $V \otimes \mathbb{Q}_{l}^{\text {ac }}$ of $G$. We write $\mathbb{Q}_{l}^{a c}(1)$ for the $l$-adic representation of $G$ defined by the character $\nu$. Thus, we have $V_{0}=V \otimes \mathbb{Q}_{l}^{\text {ac }}(-1)$. For any integer $s \geq 1$, let $\Lambda^{\bullet} V_{0}^{s}$ be the exterior algebra of the sum of $s$ copies of $V_{0}$. (In the following, we also allow $s=0$ in which case $\wedge^{\bullet} V_{0}^{0}$ denotes the trivial representation $\mathbb{Q}_{l}^{\text {ac }}$.) These $l$-adic representations of $G$ play a special role in the theory, since the associated lisse étale sheaves have a natural geometric interpretation, as described below.

4.1.1 Let $K$ be a sufficiently small open compact subgroup of $G\left(\mathbb{A}^{\infty}\right)$. We denote by $\alpha_{K}: A_{K} \rightarrow \mathrm{Sh}_{K}$ the universal abelian scheme over $\mathrm{Sh}_{K}$, and by $\alpha_{K}^{s}:$ $A_{K}^{s} \rightarrow \mathrm{Sh}_{K}$ its $s$-fold self-product over $\mathrm{Sh}_{K}$, for all $s \geq 1$. (In the following, we also allow $s=0$ in which case by $A_{K}^{0}$ we simply mean $\mathrm{Sh}_{K}$. We also sometime omit the index $K$ from the notations.) We define $\mathcal{L}=\mathcal{L}_{K}=R^{1} \alpha_{K *} \mathbb{Q}_{l}^{\text {ac }}$. Then, $\mathcal{L}$ is a lisse étale sheaf over $\mathrm{Sh}_{K}$, and more precisely it is the lisse étale sheaf associated with the representation $V_{0}$. More generally, for any integers $s, t \geq 0$, the sheaf $\mathcal{L}_{s, t}=\mathcal{L}_{s, t, K}=R^{t} \alpha_{K *}^{s} \mathbb{Q}_{l}^{\text {ac }}$ over $S h_{K}$ is the lisse étale sheaf associated with the representation $\wedge^{t} V_{0}^{s}$.

4.1.2 For any integer $s \geq 0$, let $E_{s}$ be the $\mathbb{Q}_{l}^{\text {ac }}$-algebra $\operatorname{End}\left(\wedge^{\bullet} V_{0}^{s}\right)$. We recall that an element $u \in E_{s}$ is said to be homogeneous of degree $t$ if its image is contained in $\wedge^{t} V_{0}^{s} \subset \wedge^{\bullet} V_{0}^{s}$. Weyl's theorem on complete reducibility implies that every finite dimensional representation of $G$ over $\mathbb{Q}_{l}^{\text {ac }}$ is completely reducible. Furthermore, in our context, every irreducible representation of $G$ appears in an exterior algebra of a sum of copies of $V_{0}$. ([5], Section 15.3, Proposition 15.15 for type $A_{n-1}$; Section 17.3, Theorems 17.11, 17.21 for type $C_{n}$; Section 19.5, Theorems $19.19,19.22$ for type $D_{n}$. In loc. cit., these results are stated for isometry groups but can be adapted to similitude groups.) More precisely, for any irreducible representation $\xi$ of $G$ on a finite dimensional $\mathbb{Q}_{l}^{\text {ac }}$-vector space $W_{\xi}$, there exist two integers $s=s_{\xi} \geq 0$, $t=t_{\xi} \geq 0$ and an idempotent $\epsilon=\epsilon_{\xi} \in E_{s}$ such that $\epsilon$ is homogeneous of degree $t$, and

$$
W_{\xi}=\epsilon\left(\wedge^{\bullet} V_{0}^{s}\right)
$$

For each $\xi$, the idempotent $\epsilon_{\xi}$ lies in the subalgebra of $E_{s}, s=s_{\xi}$, generated by $\operatorname{End}\left(V_{0}^{s}\right)$ and certain endomorphisms of $\wedge^{\bullet} V_{0}^{s}$ associated with the bilinear form on $V$, regarded as an element in $\wedge^{2} V_{0}^{2}(1)$.

4.1.3 We deduce that, for each irreducible finite dimensional representation $\xi$ of $G$, there exists a natural isomorphism of lisse étale sheaves over $\mathrm{Sh}_{K}$

$$
\mathcal{L}_{\xi} \simeq \epsilon_{\xi}\left(R^{t_{\xi}} \alpha_{*}^{s_{\xi}} \mathbb{Q}_{l}^{\mathrm{ac}}\right)
$$

and moreover $\epsilon_{\xi}\left(R^{t} \alpha_{*}^{s_{\xi}} \mathbb{Q}_{l}^{\text {ac }}\right)=0$ for all $t \neq t_{\xi}$. Furthermore, for each irreducible representation $\xi$, the action of the associated idempotent $\epsilon_{\xi} \in E_{s}$ on the sheaves $R^{t} \alpha_{*}^{s} \mathbb{Q}_{l}^{\text {ac }}$, for $t \geq 0, s=s_{\xi}$, has a natural geometric interpretation. It can be defined 
using the endomorphism structure, polarizations, permutations and multiplication by scalars on the $s$-fold self-product of the universal abelian scheme. This observation justifies what follows in Sections 4.2 and 4.3 where these idempotents are used in both the generic and special fibers.

Proposition 4.1 Maintaining the above notations. For each $i \geq 0$, there exists a canonical Galois equivariant isomorphism of $\mathbb{Q}_{l}^{a c}$-spaces

$$
H_{c}^{i}\left(S h_{K} \times_{E} E^{a c}, \mathcal{L}_{\xi}\right) \simeq \epsilon_{\xi} H_{c}^{i+t_{\xi}}\left(A_{K}^{s_{\xi}} \times_{E} E^{a c}, \mathbb{Q}_{l}^{a c}\right)
$$

and an equality of virtual admissible/continuous $G\left(\mathbb{A}^{\infty}\right) \times G a l\left(E^{a c} / E\right)$-modules

$$
H_{c}\left(S h, \mathcal{L}_{\xi}\right)=(-1)^{t_{\xi}} \epsilon_{\xi} H_{c}\left(A^{s_{\xi}}, \mathbb{Q}_{l}\right),
$$

where $H_{c}\left(A^{s_{\xi}}, \mathbb{Q}_{l}\right)=\sum_{i}(-1)^{i} \lim _{K} H_{c}^{i}\left(A_{K}^{s_{\xi}} \times_{E} E^{a c}, \mathbb{Q}_{l}\left(t_{\xi}\right)\right)$.

Proof The proposition is an immediate consequence of the aforementioned properties of the idempotents $\epsilon_{\xi}$ and the properness of $\alpha_{K}: A_{K} \rightarrow \mathrm{Sh}_{K}$.

4.2 Cohomology groups with coefficients in $\mathcal{L}_{\xi}$. In the following, we proceed to adapt the previous observations to the context of the modules associated with the cohomology groups of the reduction of the Shimura varieties, of their Newton strata and of the Igusa varieties.

4.2.1 Let $K$ be an open compact subgroup of $G\left(\mathbb{A}^{\infty}\right)$ as in Section 2.1.3. For each $s \geq 0$, we write $A_{K}^{s}$ for the $s$-fold self product of the universal abelian scheme over $S_{K}$, regarded as a scheme over $\mathcal{O}_{E_{v}}$. We denote by $\bar{A}_{K}^{s}$ its reduction in positive characteristic $p$, i.e. $\bar{A}_{K}^{s}=A_{K}^{s} \times_{\operatorname{Spec}\left(\mathcal{O}_{E_{v}}\right)} \operatorname{Spec}(k)$, and by $R \Psi_{\eta}^{A}=R \Psi_{\eta}^{A_{K}^{s}}$ its vanishing cycle functor. For all $p, q \geq 0$, the spaces

$$
H_{c}^{p}\left(\bar{A}^{s} \times_{k} k^{\mathrm{ac}}, R^{q} \Psi_{\eta}^{A}\left(\mathbb{Q}_{l}^{\mathrm{ac}}\right)\right)=\lim _{K} H_{c}^{p}\left(\bar{A}_{K}^{s} \times_{k} k^{\mathrm{ac}}, R^{q} \Psi_{\eta}^{A}\left(\mathbb{Q}_{l}^{\mathrm{ac}}\right)\right)
$$

are admissible/continuous $G\left(\mathbb{A}^{\infty}\right)^{\dagger} \times W_{E_{v}}$-modules, and we define

$$
H_{c}\left(\bar{A}^{s} \times_{k} k^{\mathrm{ac}}, R \Psi_{\eta}^{A}\left(\mathbb{Q}_{l}^{\mathrm{ac}}\right)\right)=\sum_{p, q}(-1)^{p+q} H_{c}^{p}\left(\bar{A}^{s} \times_{k} k^{\mathrm{ac}}, R^{q} \Psi_{\eta}^{A}\left(\mathbb{Q}_{l}^{\mathrm{ac}}\right)\right) .
$$

Proposition 4.2 Let $\xi$ be an irreducible finite dimensional $l$-adic representation of $G$. For each level $K$, there is an equality of virtual continuous $W_{E_{v}}$-modules

$$
H_{c}\left(\bar{S}_{K} \times_{k} k^{a c}, R \Psi_{\eta}^{S}\left(\mathcal{L}_{\xi}\right)\right)=(-1)^{t_{\xi}} \epsilon_{\xi} H_{c}\left(\bar{A}_{K}^{s_{\xi}} \times_{k} k^{\mathrm{ac}}, R \Psi_{\eta}^{A}\left(\mathbb{Q}_{l}^{a c}\right)\right) .
$$

Further more, there is an equality of virtual admissible/continuous $G\left(\mathbb{A}^{\infty}\right)^{\dagger} \times W_{E_{v}}$ modules

$$
H_{c}\left(\bar{S} \times_{k} k^{a c}, R \Psi_{\eta}^{S}\left(\mathcal{L}_{\xi}\right)\right)=(-1)^{t_{\xi}} \epsilon_{\xi} H_{c}\left(\bar{A}^{s_{\xi}} \times_{k} k^{\mathrm{ac}}, R \Psi_{\eta}^{A}\left(\mathbb{Q}_{l}^{a c}\right)\right) .
$$

Proof In order to deduce the latter equalities from the above constructions, it suffices to recall that the vanishing cycle functor and the derived pushforward by a proper morphism commute ([4], Section 2.1.7, p. 20). We deduce that, for all integers $s \geq 0$, there is a canonical quasi-isomorphism of derived functors

$$
R \Psi_{\eta}^{S} \circ R\left(\alpha_{\eta}^{s}\right)_{*} \simeq R\left(\bar{\alpha}^{s}\right)_{*} \circ R \Psi_{\eta}^{A^{s}}
$$

where $\alpha_{\eta}^{s}$ and $\bar{\alpha}^{s}$ denote respectively the restrictions of $\alpha^{s}: A^{s} \rightarrow S$ to the generic and special fibers of $\mathcal{O}_{E_{v}}$ (here, $\alpha^{0}=\mathrm{id}_{S}$ ). 
4.2.2 Let $b \in B\left(G_{\mathbb{Q}_{p}}, \mu_{\mathbb{Q}_{p}}\right)$. For all levels $K$ and integers $s \geq 0$, we write $\bar{A}_{K}^{s}(b)$ for the restriction of $\bar{A}_{K}^{s} / \bar{S}_{K}$ to the Newton stratum $\bar{S}_{K}(b)$, and with definition analogous to those above, introduce the $W_{E_{v}}$-module $H_{c}\left(\bar{A}_{K}^{s}(b) \times_{k}\right.$ $\left.k^{\text {ac }}, R \Psi_{\eta}^{A}\left(\mathbb{Q}_{l}^{\text {ac }}\right)\right)$ and the admissible/continuous $G\left(\mathbb{A}^{\infty}\right)^{\dagger} \times W_{E_{v}}$-module $H_{c}\left(\bar{A}^{s}(b) \times_{k}\right.$ $\left.k^{\text {ac }}, R \Psi_{\eta}^{A}\left(\mathbb{Q}_{l}^{\text {ac }}\right)\right)$. They are endowed with a natural action of the idempotents $\epsilon_{\xi}$, for all $\xi$ with $s=s_{\xi}$.

Proposition 4.3 Let $b \in B\left(G_{\mathbb{Q}_{p}}, \mu_{\mathbb{Q}_{p}^{a c}}\right)$, and $\xi$ an irreducible finite dimensional $l$-adic representation of $G$. For each level $K$, there is an equality of virtual continuous $W_{E_{v}}$-modules

$$
H_{c}\left(\bar{S}_{K}(b) \times_{k} k^{a c}, R \Psi_{\eta}^{S}\left(\mathcal{L}_{\xi}\right)\right)=(-1)^{t_{\xi}} \epsilon_{\xi} H_{c}\left(\bar{A}_{K}^{s_{\xi}}(b) \times_{k} k^{\mathrm{ac}}, R \Psi_{\eta}^{A}\left(\mathbb{Q}_{l}^{a c}\right)\right) .
$$

Further more, there is an equality of virtual admissible/continuous $G\left(\mathbb{A}^{\infty}\right)^{\dagger} \times W_{E_{v}}{ }^{-}$ modules

$$
H_{c}\left(\bar{S}(b) \times_{k} k^{a c}, R \Psi_{\eta}^{S}\left(\mathcal{L}_{\xi}\right)\right)=(-1)^{t_{\xi}} \epsilon_{\xi} H_{c}\left(\bar{A}^{s_{\xi}}(b) \times_{k} k^{\mathrm{ac}}, R \Psi_{\eta}^{A}\left(\mathbb{Q}_{l}^{a c}\right)\right) .
$$

Proof This is an immediate consequence of the definition of the idempotents $\epsilon_{\xi}$, proposition 4.2 and the Proper Base Change Theorem.

4.2.3 Let $K$ be of the form $K=K^{p} K_{p, 0}$. We assume that the Newton stratum $\bar{S}_{K}^{p}(b)$ is not empty, and let $\mathbb{X}$ be a Barsotti-Tate group with additional structures associated with a closed geometric point of $\bar{S}_{K^{p}}(b)$. For each $m \geq 1$, we write $\operatorname{Ig}_{\mathbb{X}, K^{p}, m}$ for the associated Igusa variety of level $m$ over $\bar{S}_{K^{p}}(b)$, and $A_{b, K^{p}}^{s}=$ $A_{\mathbb{X}, K^{p}, m}^{S}$ for the pullback of the $s$-fold self-product of universal abelian scheme $\bar{A}_{K^{p}}(b) / \bar{S}_{K^{p}}(b)$ over $\operatorname{Ig}_{K^{p}, m}$, for all $s \geq 0$. For all $i \geq 0$, the spaces

$$
H_{c}^{i}\left(A_{b}^{s}, \mathbb{Q}_{l}^{\mathrm{ac}}\right)=\lim _{\longrightarrow} K^{p}, m H_{c}^{i}\left(A_{\mathbb{X}, K^{p}, m}^{s}, \mathbb{Q}_{l}^{\mathrm{ac}}\right)
$$

are admissible/continuous $G\left(\mathbb{A}^{\infty, p}\right) \times J_{b}\left(\mathbb{Q}_{p}\right)$-modules, and we define

$$
H_{c}\left(A_{b}^{s}, \mathbb{Q}_{l}^{\mathrm{ac}}\right)=\sum_{i}(-1)^{i} H_{c}^{i}\left(A_{b}^{s}, \mathbb{Q}_{l}^{\mathrm{ac}}\right) .
$$

Proposition 4.4 Let $b \in B\left(G_{\mathbb{Q}_{p}}, \mu_{\mathbb{Q}_{p}^{a c}}\right)$ be an element associated with a nonempty Newton stratum, and $\xi$ an irreducible finite dimensional l-adic representation of $G$. There is an equality of virtual admissible/continuous $G\left(\mathbb{A}^{\infty, p}\right) \times J_{b}\left(\mathbb{Q}_{p}\right)$ modules

$$
H_{c}\left(I g_{b}, \mathcal{L}_{\xi}\right)=(-1)^{t_{\xi}} \epsilon_{\xi} H_{c}\left(A_{b}^{s \xi}, \mathbb{Q}_{l}^{a c}\right)
$$

Proof This is also a consequence of the definition of the idempotents $\epsilon_{\xi}$, proposition 4.2 and of the Proper Base Change Theorem.

4.3 Cohomology of the universal abelian scheme. In view of the observation of Section 4.2, we now restate Theorems 3.1 and 3.3 in the special case of the representations $\wedge^{\bullet} V_{0}^{s}, s \geq 0$, as results about the cohomology of the multi-selfproducts of the universal abelian scheme over the Shimura varieties.

Theorem 4.5 Let $s \geq 0$. For each $b \in B\left(G_{\mathbb{Q}_{p}}, \mu_{\mathbb{Q}_{p}^{a c}}\right)$ (associated with a nonempty Newton stratum), there is an equality of virtual $G\left(\mathbb{A}^{\infty}\right)^{\dagger} \times W_{E_{v}} \times S_{s}$-modules

$$
H_{c}\left(\bar{A}^{s}(b) \times_{k} k^{a c}, R \Psi_{\eta}^{A}\left(\mathbb{Q}_{l}\right)\right)=\mathcal{E}_{b}\left(H_{c}\left(A_{b}^{s}, \mathbb{Q}_{l}\right)\right),
$$

where $\mathcal{E}_{b}$ is the functor associated with the local models of the Shimura varieties attached to $b$. In the case of proper Shimura varieties, the above equalities can be 
extended to the equalities of $G\left(\mathbb{A}^{\infty}\right) \times W_{E_{v}} \times S_{s}$-modules, for all $b \in B\left(G_{\mathbb{Q}_{p}}, \mu_{\mathbb{Q}_{p}^{a c}}\right)$. In particular, we deduce an equality of $G\left(\mathbb{A}^{\infty}\right) \times W_{E_{v}} \times S_{s}$-modules

$$
H_{c}\left(A^{s}, \mathbb{Q}_{l}\right)=\sum_{b \in B\left(G_{\mathbb{Q}_{p}}, \mu_{\mathbb{Q}_{p} a c}\right)} \mathcal{E}_{b}\left(H_{c}\left(A_{b}^{s}, \mathbb{Q}_{l}\right)\right)
$$

Theorem 4.6 Let $s \geq 0, b \in B\left(G_{\mathbb{Q}_{p}}, \mu_{\mathbb{Q}_{p}^{a c}}\right)$ (associated with a non-empty Newton stratum), and $K=K^{p} K_{p}$ a level of the form $K_{p}=K_{p, m}$, for $m \geq 0$. There exists a system of $W_{E_{v}} \times S_{s}$-quasi-isomorphism of complexes, indexed by the quadruples $(N, m, n, d)$,

$$
\begin{aligned}
& \pi_{N}^{*}\left(R \Psi_{\eta}^{S} R f_{K_{p} *}\left(R \alpha_{K *}^{s}\left(\mathbb{Q}_{l}^{a c}\right)\right)_{\mid \bar{S}(b)_{k}{ }^{a c}}\right) \\
& \quad \cong q_{1}^{*}\left(R \alpha_{K^{p} *}^{s}\left(\mathbb{Q}_{l}^{a c}\right)_{\mid I_{g_{0}}}\right) \otimes q_{2}^{*}\left(R \Psi_{\eta}^{\mathcal{M}} R g_{K_{p} *}\left(\mathbb{Q}_{l}^{a c}\right)\right),
\end{aligned}
$$

which are compatible under the morphisms induced by the projections among the Igusa varieties, the inclusion of truncated Rapoport-Zink spaces and the action of $J_{b}\left(\mathbb{Q}_{p}\right)^{\dagger}$. Furthermore, they are also compatible with the action of $G\left(\mathbb{A}^{\infty}\right)^{\dagger}$, as the level $K$ varies.

Proof of Theorem 4.5 It follows from the discussion in Section 3.1 that this statement is implied by Theorem 4.6 together with Theorem 3.2. Again, in the case of proper varieties, the second equality follows from a version of Theorem 4.6 which includes the case of the vanishing cycle sheaves associated with a larger class of integral models.

Proof of Theorem 3.3 It is an immediate consequence of the properties of the idempotents $\epsilon_{\xi}$ that Theorem 4.6 implies Theorem 3.3.

4.3.1 We point that, in view of Propositions 4.1, 4.2, 4.3 and 4.4, the latter argument also proves that Theorem 3.1 follows directly from Theorem 4.5. Indeed, it suffices to observe that the action of the idempotent $\epsilon_{\xi}$ on the modules $H_{c}\left(A_{b}^{s}, \mathbb{Q}_{l}\right)$, for all $s, \xi$, commutes with the functor $\mathcal{E}_{b}$, and this follows from the fact that the actions of $G\left(\mathbb{Q}_{l}\right)$ and $J_{b}\left(\mathbb{Q}_{p}\right)$ commute.

\section{Proof of Theorem 4.6}

5.1 Preliminaries. Let $s \geq 0, K^{p} \subset G\left(\mathbb{A}^{\infty, p}\right), b \in B\left(G_{\mathbb{Q}_{p}}, \mu_{\mathbb{Q}_{p}}\right)$ and $\mathbb{X}$ a Barsotti-Tate group associated with $b$ be all as above. We write

$$
\bar{\alpha}=\bar{\alpha}_{K^{p}}: \bar{A}_{K^{p}}^{s}(b) \rightarrow \bar{S}_{K^{p}}(b)
$$

for the multi-self-product of the universal abelian scheme over the Newton stratum associated with $b$, and

$$
\beta=\beta_{K^{p}}: A_{b, K^{p}}^{s} \rightarrow \operatorname{Ig}_{b, K^{p}, 0}=\operatorname{Ig}_{\mathrm{X}, K^{p}, 0}
$$

for the pullback of $\bar{A}_{K^{p}}^{s}(b) \rightarrow \bar{S}_{K^{p}}(b)$ over the corresponding Igusa variety of level 0 , under the natural inclusion $\operatorname{Ig}_{b, K^{p}, 0} \subset \bar{S}_{K^{p}}(b)$. (For simplicity, we are here omitting the upper index $s$ in the notation of the structure morphisms, differently than in Section 4.) 
5.1.1 Let $m, n, d, N$ be non-negative integers as in Section 3.1.1. We write $\operatorname{Ig}_{b, K^{p}, m}=\operatorname{Ig}_{\mathbb{X}, K^{p}, m}$, and $\overline{\mathcal{M}}_{b}^{n, d}$ for the reduced closed fiber of the truncated Rapoport-Zink space $\mathcal{M}_{b, \mathbb{X}}^{n, d}$. We introduce two distinct abelian schemes defined over the product $\operatorname{Ig}_{b, K^{p}, m} \times \overline{\mathcal{M}}_{b}^{n, d}$. The first one is the pullback of the abelian scheme $A_{b, K^{p}}^{s} \rightarrow \operatorname{Ig}_{b, K^{p}, 0}$ under the projection $q_{1}: \operatorname{Ig}_{b, K^{p}, m} \times \overline{\mathcal{M}}_{b, K_{p}, 0}^{n, d} \rightarrow \operatorname{Ig}_{b, K^{p}, 0}$, we denote it by

$$
\gamma=\gamma_{K^{p}}=\beta \times 1: \mathcal{A}_{b, K^{p}}^{s}=q_{1}^{*} A_{b, K^{p}}^{s}=A_{b, K^{p}}^{s} \times \overline{\mathcal{M}}_{b}^{n, d} \rightarrow \operatorname{Ig}_{b, K^{p}, m} \times \overline{\mathcal{M}}_{b}^{n, d} .
$$

The second one is the pullback of $\bar{A}_{K^{p}}^{s}(b) \rightarrow \bar{S}_{K^{p}}(b)$ under the map $\pi_{N}=\pi_{(N, m, n, d)}$ introduced in Section 3.1.1, we write

$$
\delta=\delta_{K^{p}, N}: \mathcal{B}_{b, K^{p}, N}^{s}=\pi_{N}^{*} \bar{A}_{K^{p}}^{s}(b) \rightarrow \operatorname{Ig}_{b, K^{p}, m} \times \overline{\mathcal{M}}_{b}^{n, d} .
$$

In the following, with abuse of notation, we also denote by $\pi_{N}$ the corresponding morphism $\mathcal{B}_{b, K^{p}, N}^{s} \rightarrow \bar{A}_{K^{p}}^{s}(b)$. We remark that, given the moduli interpretation of the scheme $\bar{S}_{K^{p}}(b)$, the datum of the abelian scheme $\mathcal{B}_{b, K^{p}, N}$ over $\operatorname{Ig}_{b, K^{p}, m} \times \overline{\mathcal{M}}_{b}^{n, d}$ is equivalent to the datum of the corresponding morphism $\pi_{N}$. We will recall the explicit definition of the schemes $\mathcal{B}_{b, K^{p}, N}$ in the proof of proposition 5.2.

5.1.2 To extend the previous definitions to the cases of general levels $K$, of the form $K=K^{p} K_{p}$ as in Section 2.1.3, we consider the morphisms $1 \times g_{K_{p}}$ : $\operatorname{Ig}_{b, K^{p}, m} \times \overline{\mathcal{M}}_{b, K_{p}}^{n, d} \rightarrow \operatorname{Ig}_{b, K^{p}, m} \times \overline{\mathcal{M}}_{b}^{n, d}$, where $g_{K_{p}}: \overline{\mathcal{M}}_{b, K_{p}}^{n, d} \rightarrow \overline{\mathcal{M}}_{b}^{n, d}$ denote the natural projections associated with the change of level. (For each $n, d$, we denote by $\overline{\mathcal{M}}_{b, K_{p}}^{n, d}$ the pullback over $\overline{\mathcal{M}}_{b}^{n, d}$ of the cover $\overline{\mathcal{M}}_{b, K_{p}} \rightarrow \overline{\mathcal{M}}_{b}$.) For each level $K$, we define

$$
\gamma_{K}: \mathcal{A}_{b, K}^{s}=A_{b, K^{p}}^{s} \times \overline{\mathcal{M}}_{b, K_{p}}^{n, d} \rightarrow \operatorname{Ig}_{b, K^{p}, m} \times \overline{\mathcal{M}}_{b, K_{p}}^{n, d}
$$

and

$$
\delta_{K}=\delta_{K, N}: \mathcal{B}_{b, K}^{s} \rightarrow \operatorname{Ig}_{b, K^{p}, m} \times \overline{\mathcal{M}}_{b, K_{p}}^{n, d}
$$

respectively as the pullbacks of $\gamma_{K^{p}}$ and $\delta_{K^{p}, N}$ under the map $1 \times g_{K_{p}}$. With abuse of notations, we denote the natural projections $\mathcal{A}_{b, K}^{s} \rightarrow \mathcal{A}_{b, K^{p}}^{s}$ and $\mathcal{B}_{b, K}^{s} \rightarrow \mathcal{B}_{b, K^{p}}^{s}$ also by $g_{K_{p}}$. We recall that, for all level $K, \bar{\alpha}_{K}: \bar{A}_{K}^{s}(b) \rightarrow \bar{S}_{K}(b)$ denotes the pullback of $\bar{\alpha}=\bar{\alpha}_{K^{p}}$ under the map $f_{K_{p}}: \bar{S}_{K}(b) \rightarrow \bar{S}_{K^{p}}(b)$. In the following, we also write $f_{K_{p}}$ for the associated projection $\bar{A}_{K}^{s}(b) \rightarrow \bar{A}_{K^{p}}^{s}(b)$.

5.1.3 We observe that, for all levels $K$, the schemes $\bar{A}_{K}^{s}(b)$ arise as the special fiber of integral abelian schemes, namely (the restriction of) the corresponding multi-self-products of the universal abelian schemes over the integral models of the Shimura varieties. The same does not hold for the abelian schemes $\mathcal{A}_{b, K}^{s}$ and $\mathcal{B}_{b, K}^{s}$, which are defined only in positive characteristic. In particular, while over $\bar{A}_{K}^{s}(b)$ it is only natural to consider the vanishing cycle sheaves associated with the corresponding integral abelian schemes in characteristic zero, the analogous construction is not available over $\mathcal{A}_{b, K}^{s}$ and $\mathcal{B}_{b, K}^{s}$. Instead, we consider the pullbacks over $\mathcal{A}_{b, K}^{s}$ and $\mathcal{B}_{b, K}^{s}$ of the vanishing cycle sheaves associated with the RapoportZink space of the same level. To explain such a choice, we observe that if appropriate integral abelian schemes existed (and indeed, Serre-Tate theorem implies that the datum of the universal Barsotti-Tate group over $\mathcal{M}_{b, K}$ formally locally defines such lifts), then the associated vanishing cycle sheaves over $\mathcal{A}_{b, K}^{s}$ and $\mathcal{B}_{b, K}^{s}$ would 
tautologically agree with pullbacks of those associated with the Rapoport-Zink spaces.

5.2 Proof. We deduce Theorem 4.6 from the following two results.

Proposition 5.1 Maintaining the above notations. There is a system of $W_{E_{v}} \times$ $S_{s}$-quasi-isomorphisms of complexes

$$
\pi_{N}^{*}\left(R \Psi_{\eta}^{S} R f_{K_{p} *}\left(R \alpha_{K *}\left(\mathbb{Q}_{l}^{a c}\right)\right)_{\mid \bar{S}(b)_{k^{a c}}}\right) \cong R \delta_{N *} \delta_{N}^{*}\left(q_{2}^{*} R \Psi_{\eta}^{\mathcal{M}} R g_{K_{p} *}\left(\mathbb{Q}_{l}^{a c}\right)\right),
$$

indexed by quadruples $(N, m, n, d)$, such that, as $(N, m, n, d)$ varies, they are compatible under the pullback by the projections among the Igusa varieties, the inclusion among the truncated Rapoport-Zink spaces, and the action of $J_{b}\left(\mathbb{Q}_{p}\right)^{\dagger}$. They are also compatible under pullback by the action of $G\left(\mathbb{Q}_{p}\right)^{\dagger}$, as the level $K$ varies.

Proposition 5.2 Maintaining the above notations. There is a system of $W_{E_{v}} \times$ $S_{s}$-quasi-isomorphisms of complexes

$$
R \delta_{N *} \delta_{N}^{*}\left(q_{2}^{*} R \Psi_{\eta}^{\mathcal{M}} R g_{K_{p} *}\left(\mathbb{Q}_{l}^{a c}\right)\right) \cong q_{1}^{*}\left(R \alpha_{K^{p} *}\left(\mathbb{Q}_{l}^{a c}\right)_{\mid I g_{0}}\right) \otimes q_{2}^{*}\left(R \Psi_{\eta}^{\mathcal{M}} R g_{K_{p} *}\left(\mathbb{Q}_{l}^{a c}\right)\right),
$$

indexed by quadruples $(N, m, n, d)$, such that, as $(N, m, n, d)$ varies, they are compatible under the pullback by the projections among the Igusa varieties, the inclusion among the truncated Rapoport-Zink spaces, and the action of $J_{b}\left(\mathbb{Q}_{p}\right)^{\dagger}$. They are also compatible under pullback by the action of $G\left(\mathbb{Q}_{p}\right)^{\dagger}$, as the level $K$ varies.

Proof of Theorem 4.6 The system of quasi-isomorphisms in the statement is the composition of those appearing in Propositions 5.1 and 5.2.

Proof of proposition 5.1 It follows from the fact that the vanishing cycle functor commutes with derived pushforward ([1], Corollary 4.2, p.548; [2], Corollary 2.3 , p. 373) that

$$
\begin{aligned}
\pi_{N}^{*}\left(R \Psi_{\eta}^{S} R f_{K_{p} *}\left(R \alpha_{K *}\left(\mathbb{Q}_{l}^{\mathrm{ac}}\right)\right)\right) & \cong \pi_{N}^{*}\left(R \Psi_{\eta}^{S} R \alpha_{K^{p} *}\left(R f_{K_{p} *}\left(\mathbb{Q}_{l}^{\mathrm{ac}}\right)\right)\right) \\
& \cong \pi_{N}^{*}\left(R \bar{\alpha}_{K^{p} *} R \Psi_{\eta}^{A}\left(R f_{K_{p} *}\left(\mathbb{Q}_{l}^{\mathrm{ac}}\right)\right)\right),
\end{aligned}
$$

and from the Proper Base Change Theorem ([14], Corollary 2.3, pp. 223-224) that

$$
\pi_{N}^{*}\left(R \bar{\alpha}_{K^{p} *} R \Psi_{\eta}^{A}\left(R f_{K_{p} *}\left(\mathbb{Q}_{l}^{\mathrm{ac}}\right)\right)\right) \cong R \delta_{N, K^{p} *}\left(\pi_{N}^{*} R \Psi_{\eta}^{A}\left(R f_{K_{p} *}\left(\mathbb{Q}_{l}^{\mathrm{ac}}\right)\right)\right)
$$

(Here above, and through out the rest of the proof, for $\mathcal{L}$ a sheaf defined over $\bar{S}_{K^{p}} \times$ $k^{\text {ac }}$, we write $\pi_{N}^{*} \mathcal{L}$ to mean $\pi_{N}^{*}\left(\mathcal{L}_{\mid \bar{S}(b)_{k^{a c}}}\right)$, where $\bar{S}(b)_{k^{\text {ac }}}=\bar{S}_{K^{p}}(b) \times k^{\text {ac }} \subset \bar{S}_{K^{p}} \times$ $k^{\text {ac }}$.) Let $q_{2}^{*} R \Psi_{\eta}^{\mathcal{M}}\left(R g_{K_{p} *}\left(\mathbb{Q}_{l}^{\mathrm{ac}}\right)\right) \cong \pi_{N}^{*} R \Psi_{\eta}^{S}\left(R f_{K_{p} *}\left(\mathbb{Q}_{l}^{\mathrm{ac}}\right)\right)$ denote the compatible system of $W_{E_{v}}$-quasi-isomorphisms in Theorem 3 ([13], Proposition 21, p. 605). It yields a system of quasi-isomorphisms

$$
R \delta_{N *} \delta_{N}^{*}\left(q_{2}^{*} R \Psi_{\eta}^{\mathcal{M}} R g_{K_{p} *}\left(\mathbb{Q}_{l}^{\mathrm{ac}}\right)\right) \cong R \delta_{N *} \delta_{N}^{*}\left(\pi_{N}^{*} R \Psi_{\eta}^{S}\left(R f_{K_{p} *}\left(\mathbb{Q}_{l}^{\mathrm{ac}}\right)\right)\right)
$$

On the other hand, the equality $\pi_{N} \circ \delta_{N}=\bar{\alpha} \circ \pi_{N}$ implies

$$
R \delta_{N *} \delta_{N}^{*}\left(\pi_{N}^{*} R \Psi_{\eta}^{S}\left(R f_{K_{p} *}\left(\mathbb{Q}_{l}^{\mathrm{ac}}\right)\right)\right) \cong R \delta_{N *}\left(\pi_{N}^{*} \bar{\alpha}_{K_{p}}^{*} R \Psi_{\eta}^{S}\left(R f_{K_{p} *}\left(\mathbb{Q}_{l}^{\mathrm{ac}}\right)\right)\right) \text {. }
$$

Thus, in order to conclude, it suffices to prove

$$
\left.R \Psi_{\eta}^{A}\left(R f_{K_{p} *}\left(\mathbb{Q}_{l}^{a c}\right)\right) \cong \bar{\alpha}_{K_{p}}^{*} R \Psi_{\eta}^{S}\left(R f_{K_{p} *}\left(\mathbb{Q}_{l}^{a c}\right)\right)\right)
$$

We recall that given a morphism of $W\left(k^{\text {ac }}\right)$-formal schemes which are locally of finite type, if the morphism is smooth then the associated map between the vanishing cycles of the trivial $l$-adic sheaf is a quasi-isomorphism ([12], Proposition 2.26, p. 235 ; $[7]$, Lemma II.5.6, p.47). We apply this result to the (smooth) $\mathcal{O}_{E_{v}}$-morphisms 
$\alpha_{K}: A_{K}^{s} \rightarrow S_{K}$, for all levels $K$ (not just $K=K^{p} K_{p, 0}$ ). By combining this with the pertinent instances of the Proper Base Change Theorem and the fact that the vanishing cycle functor commutes with derived pushforward, we obtain the following chain of quasi-isomorphisms

$$
\begin{aligned}
R \Psi_{\eta}^{A}\left(R f_{K_{p} *}\left(\mathbb{Q}_{l}^{\mathrm{ac}}\right)\right) & \cong R f_{K_{p} *}\left(R \Psi_{\eta}^{A_{K}}\left(\mathbb{Q}_{l}^{\mathrm{ac}}\right)\right) \cong R f_{K_{p} *}\left(\bar{\alpha}_{K}^{*} R \Psi_{\eta}^{S_{K}}\left(\mathbb{Q}_{l}^{\mathrm{ac}}\right)\right) \\
& \cong \bar{\alpha}_{K^{p}}^{*} R f_{K_{p} *}\left(R \Psi_{\eta}^{S_{K}}\left(\mathbb{Q}_{l}^{\mathrm{ac}}\right)\right) \cong \bar{\alpha}_{K^{p}}^{*} R \Psi_{\eta}^{S}\left(R f_{K_{p} *}\left(\mathbb{Q}_{l}^{\mathrm{ac}}\right)\right) .
\end{aligned}
$$

Finally, it is an immediate consequence of the functoriality of the above constructions and of the compatibility among the quasi-isomorphisms in Theorem 3 that the resulting system of quasi-isomorphisms, as $N, m, n, d$ and $K$ vary, has the required properties. We remark that given the general statement of Theorem 3 ([13], Proposition 21, p. 605), this proof holds also for the vanishing cycle sheaves associated with the larger class of integral models considered in [13].

Proof of Proposition 5.2 We deduce from the Künneth formula, applied to the morphism $\gamma=\beta \times 1$, that

$$
\begin{aligned}
q_{1}^{*}\left(R \alpha_{K^{p} *}\left(\mathbb{Q}_{l}^{\mathrm{ac}}\right)_{\mid \mathrm{Ig}_{0}}\right) \otimes q_{2}^{*}\left(R \Psi_{\eta}^{\mathcal{M}} R g_{K_{p} *}\left(\mathbb{Q}_{l}^{\mathrm{ac}}\right)\right) \cong \\
\cong q_{1}^{*}\left(R \beta_{K^{p} *}\left(\mathbb{Q}_{l}^{\mathrm{ac}}\right)\right) \otimes q_{2}^{*}\left(R \Psi_{\eta}^{\mathcal{M}} R g_{K_{p} *}\left(\mathbb{Q}_{l}^{\mathrm{ac}}\right)\right) \\
\cong R \gamma_{K^{p} *} \gamma_{K^{p}}^{*}\left(q_{1}^{*}\left(\mathbb{Q}_{l}^{\mathrm{ac}}\right) \otimes q_{2}^{*}\left(R \Psi_{\eta}^{\mathcal{M}} R g_{K_{p} *}\left(\mathbb{Q}_{l}^{\mathrm{ac}}\right)\right)\right) \\
\cong R \gamma_{K^{p} *} \gamma_{K^{p}}^{*}\left(q_{2}^{*}\left(R \Psi_{\eta}^{\mathcal{M}} R g_{K_{p} *}\left(\mathbb{Q}_{l}^{\mathrm{ac}}\right)\right)\right)
\end{aligned}
$$

Thus, it suffices to establish that, for any compatible system of lisse étale $l$-adic sheaves $\mathcal{F}=\mathcal{F}_{m, n, d}$, defined over the products $\operatorname{Ig}_{\mathrm{X}, K^{p}, m} \times \overline{\mathcal{M}}_{b}^{n, d}$, there exists a compatible system of quasi-isomorphisms

$$
R \delta_{N *} \delta_{N}^{*} \mathcal{F} \cong R \gamma_{*} \gamma^{*} \mathcal{F},
$$

indexed by quadruples $(N, m, n, d)$. Indeed, the existence of such a system of quasiisomorphisms applied to the sheaves $\mathcal{F}=q_{2}^{*}\left(R \Psi_{\eta}^{\mathcal{M}} R g_{K_{p} *}\left(\mathbb{Q}_{l}^{\text {ac }}\right)\right)$, for each level $K_{p}$, implies the statement. (We remark that this holds also for the vanishing cycle sheaves associated with the larger class of integral models considered in [13].) We recall that for any $K^{p}, m, n, d, N$ (with $m, N$ sufficiently large with respect to $d), \gamma: \mathcal{A}^{s} \rightarrow \operatorname{Ig}_{K^{p}, m} \times \overline{\mathcal{M}}^{n, d}$ and $\delta_{N}: \mathcal{B}_{N}^{s} \rightarrow \operatorname{Ig}_{K^{p}, m} \times \overline{\mathcal{M}}^{n, d}$ are abelian schemes constructed via two different pullbacks from the universal abelian scheme over the reduction of the corresponding Shimura variety. We remark that while $\mathcal{A}^{s}$ depends only on the indexes $K^{p}, m, n, d$, the scheme $\mathcal{B}_{N}^{s}$ depends also on a choice of a sufficiently large integer $N$. In fact, $\mathcal{B}_{N}^{s}$ is defined via pullback under the morphism $\pi_{N}=\pi_{(N, m, n, d)}$. Furthermore, as we already remarked, the datum of the morphism $\pi_{(N, m, n, d)}: \operatorname{Ig}_{K^{p}, m} \times \overline{\mathcal{M}}^{n, d} \rightarrow \bar{S}_{K^{p}}(b)$ is equivalent to that of the abelian scheme $\mathcal{B}_{N}$ over $\operatorname{Ig}_{K^{p}, m} \times \overline{\mathcal{M}}^{n, d}$. We recall the definition of $\mathcal{B}_{N}$, for any quintuple $\left(K^{p}, m, n, d, N\right)$. Let $q$ denote the cardinality of the residue field $k$ of $E$, and $F: \mathcal{A} \rightarrow \mathcal{A}^{(q)}$ the $\left(\log _{p} q\right)$-th power of the Frobenius morphism on $\mathcal{A}$. Let $\beta: \mathbb{X} \rightarrow \mathcal{H}$ denote (the pullback of) the universal quasi-isogeny over the RapoportZink space $\overline{\mathcal{M}}$, and $j_{m}: \mathbb{X}\left[p^{m}\right] \rightarrow H^{s p}\left[p^{m}\right]$ denote (the pullback of) the universal Igusa structure of level $m$ on the Barsotti-Tate group $H=\bar{A}\left[p^{\infty}\right] / C_{\mathrm{X}}$. It follows from the definition of the truncated Rapoport-Zink spaces that the restriction of $\beta$ to $\overline{\mathcal{M}}^{n, d}$ satisfies the conditions that $p^{n} \beta$ is an isogeny and $\operatorname{ker}\left(p^{n} \beta\right) \subset \mathbb{X}\left[p^{d}\right]$. Further more, in [13] (Lemma 8, p. 591-592) we proved that, given $N$ sufficiently 
large with respect to $d$ and $m \geq d$, the Igusa structure $j_{m}$ induces an isomorphisms of truncated Barsotti-Tate groups

$$
j_{m}^{\left(q^{N}\right)}=f_{N} \circ j_{m \mid \mathbb{X}\left[p^{d}\right]}: \mathbb{X}\left[p^{d}\right] \cong H^{s p}\left[p^{d}\right] \cong H^{\left(q^{N}\right)}\left[p^{d}\right]
$$

We define $\mathcal{B}_{N}=\pi_{N}^{*} \mathcal{A}$ as the quotient of $\mathcal{A}^{\left(q^{N}\right)}$ by the finite flat group scheme

$$
j_{m}^{\left(q^{N}\right)}\left(\operatorname{ker}\left(p^{n} \beta\right)\right) \subset \mathcal{A}^{\left(q^{N}\right)}\left[p^{d}\right]
$$

([13], Proposition 9, p. 592). It follows from the definition that $\mathcal{B}_{N}$ comes equipped with a $p$-power isogeny $\psi_{N}: \mathcal{A}^{\left(q^{N}\right)} \rightarrow \mathcal{B}_{N}$. We remark that it is an immediate consequence of the construction that, as the indexes $K^{p}, m, d$ vary, the corresponding pairs $\left(\mathcal{B}_{N}, \psi_{N}\right)$ agree under pullback by the projections among Igusa varieties and the inclusions among truncated Rapoport-Zink spaces. As $n$ varies, the pairs $\left(\mathcal{B}_{N}, \psi_{N}\right)$ agree under pullback only after composing $\psi_{N}$ with the appropriate power of $p$, i.e. after replacing $\psi_{N}$ by the quasi-isogeny $p^{-n} \psi_{N}$. Finally, as $N$ vary, the data $\left(\mathcal{B}_{N}, \psi_{N}\right)$ agree under pullback by the appropriate power of Frobenius, i.e. for each $N$ there is a canonical identification between $\left(\mathcal{B}_{N+1}, \psi_{N+1}\right)$ and $\left(\mathcal{B}_{N}^{(q)}, \psi_{N}^{(q)}\right)$. For any $s \geq 1$, we define $\phi_{N}=\psi_{N}^{s} \circ F^{N}: \mathcal{A}^{s} \rightarrow \mathcal{B}_{N}^{s}$. It is an easy observation that the morphisms $\phi_{N}$ are $p$-power isogenies of abelian schemes over $\operatorname{Ig}_{K^{p}, m} \times \overline{\mathcal{M}}^{n, d}$, for all indexes $K^{p}, m, n, d, N$, and that they are obviously $S_{s}$-equivariant. Let $\mathcal{F}=\mathcal{F}_{m, n, d}$ be a compatible system of lisse étale $l$-adic sheaves over the products $\operatorname{Ig}_{\mathrm{X}, K^{p}, m} \times \overline{\mathcal{M}}_{b}^{n, d}$. Then, for each set of indexes, the isogeny $\phi_{N}$ induces a morphism of complexes

$$
\phi_{N *}: R \gamma_{*} \gamma^{*} \mathcal{F} \rightarrow R \delta_{N *} \delta_{N}^{*} \mathcal{F}
$$

which are quasi-isomorphisms since $\phi_{N}$ are $p$-power isogenies and $l \neq p$. Moreover, it follows from the above discussion that after composing each $\phi_{N} *$ with $\sigma^{-N \log _{p} q} p^{-n}$, we obtain a compatible system of quasi-isomorphisms $R \gamma_{*} \gamma^{*} \mathcal{F} \cong$ $R \delta_{N *} \delta_{N}^{*} \mathcal{F}$ with the required properties (here, $\sigma$ denotes the natural action of Frobenius of $k$ on the complexes).

5.3 Remark. We conclude this paper with a brief remark aimed to clarify the proof of Theorem 4.6 and its relation to the results in [13] (namely, to Theorem 3). To do so, we explain our approach from a different point of view. Let us consider a proof of Theorem 4.6 which deals with the special case of levels $K$ which are maximal compact at $p$, i.e. $K=K^{p} K_{p, 0}$, and the general case, i.e. $K=K^{p} K_{p}$ for $K_{p} \subset K_{p, 0}$, separately. This new approach is not essentially different from our previous one. Indeed, in the case of levels $K=K^{p} K_{p, 0}$, Theorem 4.6 simply states the existence of a compatible system of $W_{E_{v}}$-quasi-isomorphism of complexes

$$
\pi_{N}^{*}\left(R \bar{\alpha}_{K^{p} *}\left(\mathbb{Q}_{l}^{\mathrm{ac}}\right)\right)_{\mid \bar{S}(b)_{k^{\mathrm{ac}}}} \cong q_{1}^{*}\left(R \bar{\alpha}_{K^{p} *}\left(\mathbb{Q}_{l}^{\mathrm{ac}}\right)_{\mid \mathrm{Ig}_{0}}\right) \otimes q_{2}^{*}\left(\mathbb{Q}_{l}^{\mathrm{ac}}\right) .
$$

This amounts to comparing the étale cohomology with constant $l$-adic coefficients of two abelian schemes in positive characteristic. The statement follows directly from the corresponding special case of proposition 5.2 and from the first half of the proof of proposition 5.1. In particular, for these levels, the pertinent vanishing cycle sheaves are trivial and the results do not rely on Theorem 3 . Subsequently, given Theorem 4.6 in the special case, in order to extend that result to the general case, we need to establish the general statements of Propositions 5.1 and 5.2. In the case of proposition 5.2 , the general statement follows immediately from its special case via Proper Base Change. The same it is not true for proposition 5.1, and in 
the second half of the proof of proposition 5.1 we rely on the theory of vanishing cycles to deduce the general statement of proposition 5.1 from Theorem 3 . In particular, the more general version of Theorem 4.6 which is required to deduce the second statement in Theorem 4.5 (under the assumption that the Shimura varieties are proper) follows directly from the corresponding version of Theorem 3 ([13], Proposition 21, p. 605).

Acknowledgment. I would like to thank R. Taylor, M. Harris, and S.W. Shin for their interest in my work and for stimulating discussions, and the referee for helpful comments and useful suggestions.

\section{References}

[1] Berkovich, V. Vanishing cycles for formal schemes. Invent. Math. 115 (1994), no. 3, 539-571.

[2] Berkovich, V. Vanishing cycles for formal schemes. II. Invent. Math. 125 (1996), no. 2, $367-390$.

[3] Berkovich, V. Étale cohomology for non-Archimedean analytic spaces. Inst. Hautes Études Sci. Publ. Math. No. 78 (1993), 5-161.

[4] Deligne, P. Le formalisme des cycles évanescents. Groupes de monodromie en géométrie algébrique. Séminaire de Géométrie Algébrique du Bois-Marie (SGA 7-II). Lecture notes in mathematics, Vol. 340, Springer-Verlag, Berlin-New York, 1972-1973.

[5] Fulton, W.; Harris, J. Representation Theory. A first course. Graduate Texts in Mathematics, 129. Readings in Mathematics. Springer-Verlag, New York, 1991.

[6] Grothendieck, A. Groupes de Barsotti-Tate et cristaux de Dieudonné. Sém. Math. Sup. Univ. Montréal. Presses Univ. Montréal, 1974.

[7] Harris, M.; Taylor, R. On the geometry and cohomology of some simple Shimura varieties. Volume 151, Annals of Math. Studies, Princeton University Press, 2001.

[8] Igusa, J. Kroneckerian model of fields of elliptic modular functions. Amer. J. Math. 81, 1959, 561-577.

[9] Katz, N.; Mazur, B. Arithmetic moduli of elliptic curves. Annals of Mathematics Studies, 108, Princeton University Press, Princeton, NJ, 1985.

[10] Kottwitz, R. Points on some Shimura varieties over finite fields. J. Amer. Math. Soc. 5 (1992), no. 2, 373-444.

[11] Kottwitz, R. Isocrystals with additional structure. Compositio Math. 56 (1985), no. 2, 201220.

[12] Mantovan, E. On certain unitary group Shimura varieties. Variétés de Shimura, espaces de Rapoport-Zink et correspondances de Langlands locales. Astérisque 291 (2004), 200-331.

[13] Mantovan, E. On the cohomology of certain PEL-type Shimura varieties. Duke Math. J. 129 (2005), no. 3, 573-610.

[14] Milne, J. S. Étale Cohomology. Princeton Mathematical Series, 33, Princeton University Press, Princeton, NJ, 1980.

[15] Oort, F. Foliations in moduli spaces of abelian varieties. J. Amer. Math. Soc. 17 (2004), no. 2, 267-296.

[16] Oort, F.; Zink, Th. Families of p-divisible groups with constant Newton polygon. Documenta Mathematica 7 (2002), 183-201.

[17] Rapoport, M.; Richartz, M. On the classification and specialization of F-isocrystals with additional structure. Compositio Math. 103 (1996), no. 2, 153-181.

[18] Rapoport, M; Zink, Th. Period spaces for p-divisible groups. Annals of Mathematics Studies, 141, Princeton University Press, Princeton, NJ, 1996.

[19] Zink, Th. On the slope filtration. Duke Math. J. Vol. 109 (2001), 79-95. 